\title{
Factorized Geometrical Autofocus for Synthetic Aperture Radar Processing
}

\author{
Jan Torgrimsson, Patrik Dammert, Senior Member, IEEE, Hans Hellsten, Member, IEEE, and \\ Lars M. H. Ulander, Senior Member, IEEE
}

\begin{abstract}
This paper describes a factorized geometrical autofocus (FGA) algorithm, specifically suitable for ultrawideband synthetic aperture radar. The strategy is integrated in a fast factorized back-projection chain and relies on varying track parameters step by step to obtain a sharp image; focus measures are provided by an object function (intensity correlation). The FGA algorithm has been successfully applied on synthetic and real (Coherent All RAdio BAnd System II) data sets, i.e., with false track parameters introduced prior to processing, to set up constrained problems involving one geometrical quantity. Resolution (3 dB in azimuth and slant range) and peak-to-sidelobe ratio measurements in FGA images are comparable with reference results (within a few percent and tenths of a decibel), demonstrating the capacity to compensate for residual space variant range cell migration. The FGA algorithm is finally also benchmarked (visually) against the phase gradient algorithm to emphasize the advantage of a geometrical autofocus approach.
\end{abstract}

Index Terms-Autofocus, back-projection, phase gradient algorithm (PGA), synthetic aperture radar (SAR).

\section{INTRODUCTION}

$\mathbf{S}$ YNTHETIC aperture radar (SAR) processing is usually performed in the frequency domain. Methods such as Fourier-Hankel inversion [1], [11], [15] and the range migration algorithm [3], [6], [23], [24] assume a linear aperture track; known deviations are then locally compensated. The validity, however, impairs, as deviations intensify. This often degrades the image.

Time-domain methods can deal with a nonlinear aperture track, presuming once again that deviations are known [27]. A brute force algorithm, i.e., global back-projection (GBP) [11], [13], [27], is straightforward to implement but normally too slow to be applied on a regular basis. Fast factorized backprojection (FFBP) [13], [27], on the other hand, has a run time in parity with the aforementioned methods; hence, the algorithm is a justified processing alternative.

Track parameters are usually measured by means of a Global Positioning System (GPS) and an inertial measurement unit

Manuscript received February 1, 2013; revised August 6, 2013 and November 26, 2013; accepted December 3, 2013. Date of publication March 5, 2014; date of current version May 22, 2014. This work was supported by the Swedish Governmental Agency for Innovation Systems (VINNOVA).

J. Torgrimsson is with Chalmers University of Technology, 41296 Gothenburg, Sweden (e-mail: jan.torgrimsson@chalmers.se).

P. Dammert and H. Hellsten are with SAAB Electronic Defence Systems, 40423 Gothenburg, Sweden (e-mail: patrik.dammert@saabgroup.com; hans.hellsten@saabgroup.com).

L. M. H. Ulander is with the Swedish Defence Research Agency (FOI), 63104 Linköping, Sweden and also with Chalmers University of Technology, 41296 Gothenburg, Sweden (e-mail: lars.ulander@foi.se).

Digital Object Identifier 10.1109/TGRS.2014.2300347
(IMU) [6], [20]. The IMU has a high frequency response but drifts over time; the GPS is used to counter this. As the IMU constitutes a major cost and is subject to export restrictions, a desire to relax requirements (or excluding it) often originates. Naturally though, measurement accuracy is an essential necessity for successful image formation. This of course contradicts the stated desire. A GPS may in addition be jammed or shadowed, leading to dependence on the IMU, again affecting the measurement accuracy.

In SAR processing, track deviations must be known within fractions $(\sim 1 / 16)$ of a wavelength [6], [20]. For high radar bands (e.g., X-band), this demand is typically too strict. Even for a low band such as very high frequency (VHF), the issues considered above may degrade the image. There is, however, a conceivable solution, making it feasible to focus an image without the otherwise necessary accuracy, viz., autofocus [16], [17].

In the context of SAR processing, autofocus is the use of information in a defocused image (or in the data) to estimate and correct phase errors [6]. Since the early 1970s, numerous parametric and nonparametric techniques have been developed. In spotlight mode, the former category includes recognized correlation routines, i.e., map drift (MD) [6], [20], multiple aperture MD [6], [20], and the phase difference algorithm [6]. Lately, a coherent MD approach has appeared in the literature as well [25]. The latter category includes the widely used phase gradient algorithm (PGA) [6], [9], [10], [19], [20], [31], which often is deemed as a superior high-order strategy. In recent years, metric-based schemes (spanning the space of parametric and nonparametric techniques) have also been gaining more and more attention, e.g., phase adjustment by contrast enhancement [21] and the minimum entropy algorithm [33].

Standard autofocus techniques are one dimensional, presuming that phase errors reside in individual range bins [6], [16], [17]. Essentially, this implies that, after SAR processing, there can be no residual range cell migration (RCM). Phase errors are, in addition, assumed invariant across the image [6], [16], [17].

In stripmap mode, standard autofocus techniques usually alleviate azimuth variant effects or phase errors (but not range variant effects), e.g., the phase curvature algorithm [30] and the stripmap PGA [26]. However, as the aperture track is not updated, the compensation is incomplete.

The preceding restrictions are not by necessity well founded [specifically not for airborne ultrawideband (UWB) SAR systems], especially not if measurement errors begin to escalate (due to relaxed requirements on the IMU/GPS and/or a 
jammed/shadowed GPS). Due to this, a number of innovative strategies have been suggested.

The 2-D PGA [14], [32] can mitigate residual space invariant RCM. Another approach is to first apply a 1-D PGA formulation on a coarse range resolution image, estimating and removing RCM prior to fine range compression. The algorithm is then applied again to correct remaining (1-D) phase errors. This strategy (and a similar semi-integrated strategy) is described in [8]. Reference [34] outlines (spotlight/stripmap) schemes based on the same idea [8] and a (stripmap) PGA implementation with a weighted maximum-likelihood (ML) kernel (as opposed to the linear unbiased minimum variance kernel in [6], [9], and [10] and the ML kernel in [19] and [20]); block processing relieves range variant effects. PGA-MD [35], in turn, relies on sub-aperture division, 2-D MD, and the (stripmap) PGA to correct residual RCM (in spotlight/stripmap mode); once again, block processing relieves range variant phase errors.

By breaking up a defocused image (or data) into space invariant areas (blocks) and processing these separately, space variant effects may be eased [6]. Naturally though, estimation accuracy deteriorates as the areas decrease in size. Additionally, border issues arise as a penalty for patching. An alternative and less abrupt (spotlight) approach, i.e., pixel-unique phase adjustment, is described in [22], but residual RCM is neglected.

It should also be stressed that multilateration techniques based on prominent point phase tracking [4] and local 2-D MD [5] do update the aperture track, providing an ability to correct residual space variant RCM. This has, however, not been demonstrated in practice.

The objective of this paper is to address aforementioned limiting premises. This can be done by formulating and testing an autofocus algorithm that compensates for the defocusing cause (measurement errors), i.e., by regulating track parameters in the time domain [16], [17]. The course of action omits reported restrictions and makes it possible to correct an inaccurate geometry from a focusing perspective.

The factorized geometrical autofocus (FGA) algorithm, which is developed within the framework of FFBP, is fully integrated in the conventional processing chain (FFBP). This implies that, just like FFBP, the FGA algorithm can be used to process spotlight or stripmap data. Basically, different geometry hypotheses (different images) are assessed; the aim is to find the sharpest image according to a chosen object function (intensity correlation in this case).

The novel strategy has been successfully applied in spotlight mode on synthetic and real data sets (real data acquired by Coherent All RAdio BAnd System II (CARABAS II) [18]), i.e., with false track parameters introduced prior to processing, to set up constrained problems involving one geometrical quantity.

In this paper, resulting images will be presented, analyzed, and compared to reference images and to images formed without autofocus, in consequence suffering from residual space variant RCM. Additionally, PGA-processed images [20] will be shown, to be able to benchmark the performance of the new algorithm.

However, before actually satisfying the stated objective, a review is required, dealing with time-domain SAR processing, the PGA, and, of course, with the FGA concept. The realization and evaluation procedure will be recapped in detail as well.

\section{MethoD}

\section{A. $G B P$}

GBP is a time-domain method, projecting pulse compressed radar echoes to a generally defined image display plane (IDP). Each slow time position (along the track) contributes with a data value to each and every pixel. Complex values are coherently added, causing interference, in turn resolving reflective structures.

The slant range between the position and the pixel coordinate in question determines which data value to accumulate; range interpolation retrieves the proper value from available samples. For demodulated data, each value must also be multiplied by a phase factor.

GBP is a versatile algorithm, i.e., dealing with nonlinear aperture tracks, topography, etc. However, the number of operations (proportional to $N^{3}$ for $N$ sample positions and an $N \times N$ image) normally restricts its use to moderately sized images [27].

\section{B. FFBP}

FFBP is a time-efficient alternative to GBP, utilizing a coherent combination scheme to merge pulse compressed radar echoes step by step. Basically, the track is partitioned into sub-apertures, increasing in length (finer angular resolution) and decreasing in number for each factorization step [27]. Every sub-aperture comes with a corresponding sub-image. The antenna beam is divided into sub-lobes, producing images with pixel coordinates in range and sub-lobe angle (indicating that the briefly mentioned scheme involves interpolation in both range and angle [13], [27]). Ideally, i.e., if the number of slow time positions is expressible as a factorization of integers, the final factorization step gives the polar aperture image. Note that, in stripmap mode, the sub-apertures may overlap to smooth transitions between individual aperture images. These are then placed side by side, registered, or resampled to a suitable representation (e.g., Cartesian).

For a base two realization, the number of operations is proportional to $2 N^{2} \log _{2} N$ (for $N$ sample positions and an $N \times N$ image), i.e., under the premise that $N$ is equal to a power of two [27]. Image quality requirements may, however, motivate a less effective algorithm execution (e.g., by reducing the number of factorization steps and/or using a more exact interpolator), i.e., to make up for the fact that interpolation errors are accumulated for each factorization step (see [13] and [27] for further information).

\section{C. $P G A$}

The PGA [20] is a notable nonparametric autofocus technique, capable of correcting phase errors of arbitrary order. The algorithm involves a number of sequential steps, typically iterated to attain convergence. First, strong targets in individual range bins are identified in a defocused image. These are 


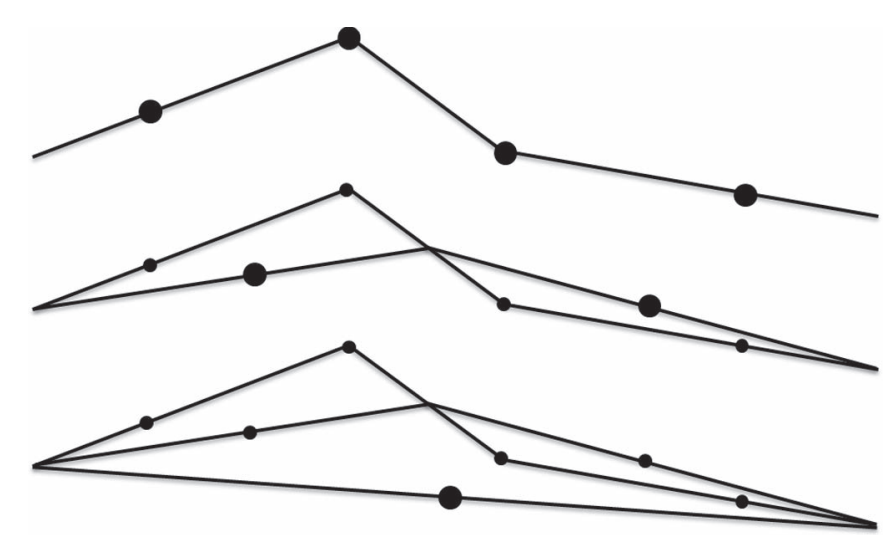

Fig. 1. (Top) Four sample positions (tied together). (Middle) After the first factorization step, two sub-apertures (and two new positions) remain. (Bottom) The second and final factorization step gives the full aperture (and one new position). In reality, naturally, the initial number of sample positions is orders of magnitude greater.

aligned through a circular shift and windowed to discard extraneous data. Next, an expression for the phase error derivative (averaged over the bins) is found in the (azimuth) frequency domain. Integrating this expression gives an estimate of the error. Range bins are multiplied by the complex conjugate of the estimate; ideally, this eliminates the phase errors. Finally, bins are transformed back to the (azimuth) time domain (the image domain).

The summarized spotlight principle (see [20] for further information) has been proven to be robust for a diversity of different scenes, and although many alternative schemes have been suggested (a routine increasing the rate of convergence is, for example, described in [7]), the conventional PGA formulation is still the standard; its vast use within the SAR community has even made it a norm for emerging autofocus strategies.

\section{FGA}

1) General Resume: To set the tone, presume that pulse compressed echoes are demodulated and factorized with base two (radar echoes merged in pairs) until two sub-apertures remain (see Fig. 1). The IDP and the focus target plane (FTP) coincide with the horizontal plane or the $x y$-plane. Despite measurement errors, the sub-images are focused; this is due to limited angular resolution. The full aperture $\left(\boldsymbol{Q}_{\mathbf{1 3}}\right)$ is synthesized as a segment (aligned with the nominal flight direction), extending from the start point $\left(p_{1}\right)$ of the first sub-aperture $\left(Q_{12}\right)$ to the endpoint $\left(p_{3}\right)$ of the other $\left(Q_{23}\right) . p_{1}, p_{3}$, and the cutoff point $\left(\boldsymbol{p}_{2}\right)$ between prior segments form a triangle (see Fig. 2) or a line as a special case. If the geometry is too inaccurate (due to measurement errors), the aperture image will be defocused.

By varying parameters defining the triangle, different geometry hypotheses can be assessed. The variation is carried out consecutively by means of a merging $(\mathrm{M})$ transform and a range history preserving (RHP) transform. In principle, pixel coordinates of the aperture image (i.e., a pixel grid with coordinates in range and sub-lobe angle) are expressed in sub-image coordinates corresponding to the current geometry. The aperture image is then found by interpolating the sub-images onto the

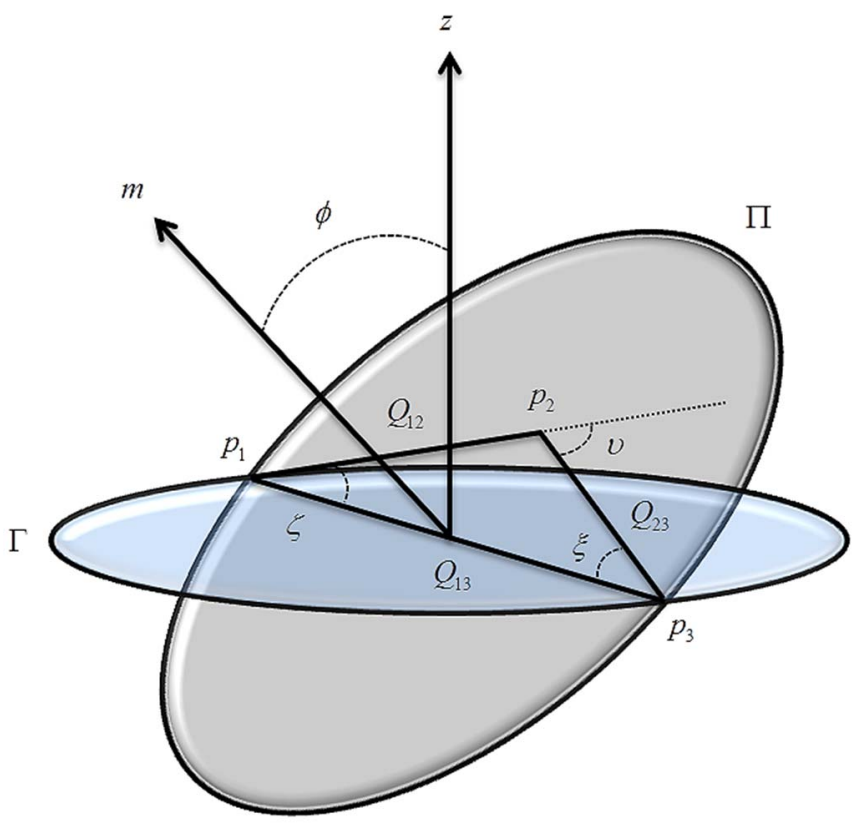

Fig. 2. Triangle in the plane $\Pi$ (gray). $\phi, v, \zeta$, and $\xi$ are essential parameters in this autofocus formulation. Note that $Q_{13}$ and a horizontal vector orthogonal to $Q_{13}$ (not shown) define the plane $\Gamma$ (blue). In this particular case, $Q_{13}$ and $\Gamma$ coincide with the $x y$-plane (blue).

polar pixel grids and adding these coherently. Fundamentally, the final factorization step is repeated time after time. Each hypothesis produces an image, which then is marked with a focus measure provided by an object function. The image with the best measure is assumed to be autofocused.

Before proceeding, it should be emphasized that, although a one-step approach is presumed, the FGA algorithm can be activated at any time during the factorization [17]. However, as the accuracy demand on track parameters increases quadratically with sub-aperture length, it is more likely that the algorithm is required later on in the processing chain.

Note also that this spotlight scheme can deal with stripmap data in the same way as FFBP does, i.e., by allowing subapertures to overlap. For example, instead of forming two images (triangles) from four sub-apertures, three images (triangles) may be formed. The two central sub-apertures are then used to produce the additional image.

Confining the variation to a number of quantities (the fewer the better) is a crucial task. In total, the triangle has nine degrees of freedom (assuming that sub-aperture segments remain connected in the cutoff point, i.e., an $x, y$, and $z$ coordinate for $p_{1}, p_{2}$, and $p_{3}$, respectively). Translating (two degrees) and rotating (one degree) the triangle horizontally will, however, only translate and rotate the aperture image. This implies that, from a focusing perspective, three degrees of freedom can be dropped. Thus, the geometry may be described by means of an altitude, three angles, and two length variables, all in all six independent quantities [17].

It should be mentioned that, in an early formulation of this algorithm [16], the height of focus is presumed to suffice, making it possible to fix the position and orientation of one subaperture $\left(\boldsymbol{Q}_{12}\right)$ and, by that, omitting two additional degrees of freedom. 


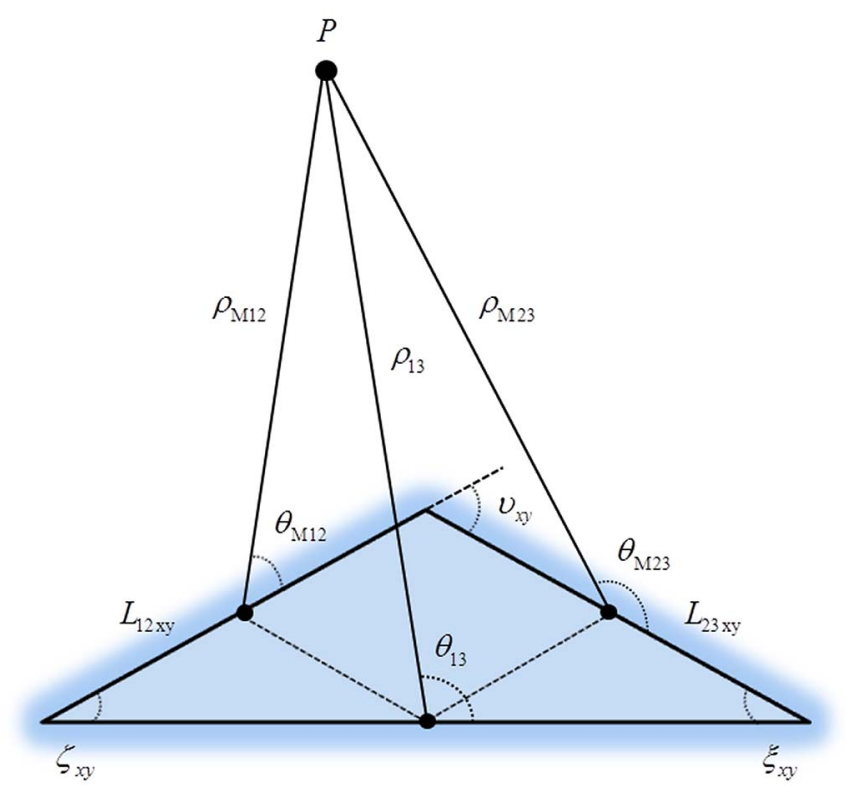

Fig. 3. Geometry for the M transform (i.e., the triangle in Fig. 2 projected to the $x y$-plane). Point $P$ is an arbitrary point in the IDP/FTP. In practice, $P$ and the vertex of the cutoff point can be located on either side of the aperture, explaining the \pm sign in (1)-(4).

2) Algorithm: Assume once again that the FGA algorithm is activated at the final factorization step. An arbitrary geometry hypothesis (contrary to the initial geometry hypothesis, given by measured track parameters) is first expressed in the following quantities (see Fig. 2 for further information):

- $H_{13}$ : altitude at the center of $\boldsymbol{Q}_{13}$;

- $\phi$ : angle between $\Gamma$ and $\Pi$;

- $\beta_{13}$ : angle between the $x y$-plane and $Q_{13}$;

- $v$ : angle between $Q_{12}$ and $Q_{23}$;

- $L_{13}$ : length of $\boldsymbol{Q}_{13}$;

- $\Delta L$ : length difference between $Q_{12}$ and $Q_{23}$.

Supporting parameters are computed, and the pixel coordinates $\left(\rho_{13}\right.$ and $\left.\theta_{13}\right)$ of the aperture image $\left(I_{13}\right)$ are established. The $\mathrm{M}$ transform then converts these to corresponding subimage coordinates. This conforms to translating and rotating sub-aperture segments horizontally, as opposed to translating and rotating an intact triangle.

Fig. 3 and (1)-(4) clarify the concept of the M transform. Note that all parameters in (1)-(4) are defined in the $x y$-plane, i.e., $L_{12 x y}$ and $L_{23 x y}$ are horizontal length variables (associated with the sub-apertures), whereas $v_{x y}, \zeta_{x y}$, and $\xi_{x y}$ are projected angles $(v, \zeta$, and $\xi)$, i.e.,

$$
\begin{aligned}
\rho_{M 12}= & \left(\rho_{13}^{2}+\left(L_{23 x y} / 2\right)^{2}-\rho_{13} \cdot L_{23 x y}\right. \\
& \left.\cdot \cos \left(\pi-\theta_{13} \pm \xi_{x y}\right)\right)^{1 / 2} \\
\theta_{M 12}= & \cos ^{-1}\left(\frac{\rho_{13}^{2}-\rho_{M 12}^{2}-\left(L_{23 x y} / 2\right)^{2}}{-\rho_{M 12} \cdot L_{23 x y}}\right) \pm v_{x y} \\
\rho_{M 23}= & \left(\rho_{13}^{2}+\left(L_{12 x y} / 2\right)^{2}-\rho_{13} \cdot L_{12 x y}\right. \\
& \left.\cdot \cos \left(\theta_{13} \pm \zeta_{x y}\right)\right)^{1 / 2} \\
\theta_{M 23}= & \pi-\cos ^{-1}\left(\frac{\rho_{13}^{2}-\rho_{M 23}^{2}-\left(L_{12 x y} / 2\right)^{2}}{-\rho_{M 23} \cdot L_{12 x y}}\right) \pm v_{x y} .
\end{aligned}
$$

M-transformed pixel coordinates are distorted by the RHP transform. This conforms to tilt, altitude, and length alterations. Contrary to the M transform, the RHP transform works with individual sub-aperture segments (note that subscripts in (5)-(11) below are dropped for clarity, i.e., either 12 or 23 , depending on the sub-aperture under consideration).

Consider $Q_{0}$, which is defined between times $-T / 2<t<$ $T / 2$ (the subscript zero signifies that $\boldsymbol{Q}_{\mathbf{0}}$ is specified by means of measured track parameters). The slant range to the point $\boldsymbol{P}$ along $Q_{0}$ as a function of time is given by

$$
\begin{array}{r}
\left\|\boldsymbol{Q}_{\mathbf{0}}(t) \boldsymbol{P}\right\|=\left(\left(\rho_{M} \cdot \sin \theta_{M}\right)^{2}+\left(\rho_{M} \cdot \cos \theta_{M}-V_{0 x y} t\right)^{2}\right. \\
\left.+\left(H_{0}+V_{0 z} t\right)^{2}\right)^{1 / 2}
\end{array}
$$

where $\rho_{M}$ is the range, whereas $\theta_{M}$ is the sub-lobe angle (in the $x y$-plane). $H_{0}$ is the altitude at the center of the subaperture, i.e., defined at time zero just as $\rho_{M}$ and $\theta_{M} . V_{0 x y}$ is the horizontal speed, whereas $V_{0 z}$ is the vertical velocity.

Now, consider $Q$, which is defined between the same times as before, but with altered horizontal speed, i.e., $V_{x y}$, vertical velocity, i.e., $V_{z}$, and altitude, i.e., $H$. The slant range to the point $\boldsymbol{P}$ along $\boldsymbol{Q}$ as a function of time is given by

$$
\begin{aligned}
\|\boldsymbol{Q}(t) \boldsymbol{P}\|=\left(\left(\rho_{M} \cdot \sin \theta_{M}\right)^{2}+\left(\rho_{M} \cdot\right.\right. & \left.\cos \theta_{M}-V_{x y} t\right)^{2} \\
& \left.+\left(H+V_{z} t\right)^{2}\right)^{1 / 2} .
\end{aligned}
$$

Note that, although $V$ components are constants in (5) and (6), this is not a limiting requirement but a way to clarify the coming derivation. In practice, $V$ components in (9)-(11) below are replaced by length, i.e., $L$, components.

The RHP transform substitutes $\rho_{M}$ and $\theta_{M}$ in (5) with primed parameters, i.e., $\rho_{M}^{\prime}$ and $\theta_{M}^{\prime}$. The goal is to find equality or at least approximate equality between the range histories of $\|\boldsymbol{Q}(t) \boldsymbol{P}\|$ and $\left\|\boldsymbol{Q}_{\mathbf{0}}^{\prime}(t) \boldsymbol{P}\right\|$. This is realized by first squaring and expanding (6) and the primed (5). Resulting polynomial coefficients $(t)$ are then set to agree, i.e.,

$$
\rho_{M}^{\prime 2}+H_{0}^{2}=\rho_{M}^{2}+H^{2} .
$$

After rearranging (7)

$$
\rho_{M}^{\prime}=\sqrt{\left(\rho_{M}^{2}+H^{2}-H_{0}^{2}\right)} .
$$

The zero-order equality in (7) is satisfied by (8), i.e.,

$$
\begin{aligned}
\left(\rho_{M}^{\prime} \cdot V_{0 x y} \cdot \cos \theta_{M}^{\prime}-\right. & \left.H_{0} \cdot V_{0 z}\right) t \\
& =\left(\rho_{M} \cdot V_{x y} \cdot \cos \theta_{M}-H \cdot V_{z}\right) t .
\end{aligned}
$$

After rearranging (9)

$$
\theta_{M}^{\prime}=\cos ^{-1}\left(\frac{\rho_{M} \cdot V_{x y} \cdot \cos \theta_{M}-H \cdot V_{z}+H_{0} \cdot V_{0 z}}{\rho_{M}^{\prime} \cdot V_{0 x y}}\right) .
$$

The first-order equality in (9) is satisfied by (10), i.e.,

$$
\left(V_{0 x y}^{2}+V_{0 z}^{2}\right) t^{2}=\left(V_{x y}^{2}+V_{z}^{2}\right) t^{2} .
$$


The second-order equality in (11) is only satisfied if the original sub-aperture length (given by the initial geometry hypothesis) is preserved while varying the geometry. However, if the sub-images are focused as assumed, second-order equality is not really required, as it is possible to compensate for measurement errors with the aid of (8) and (10) alone.

The sub-images are interpolated onto polar pixel grids with M- and RHP-transformed coordinates (corresponding to the new geometry, i.e., the arbitrary geometry hypothesis). Adding the grids coherently as in (12) then gives the aperture image. Note that phase factors (demodulated data) are omitted in (12) for clarity.

$$
I_{13}\left(\rho_{13}, \theta_{13}\right)=I_{12}\left(\rho_{M 12}^{\prime}, \theta_{M 12}^{\prime}\right)+I_{23}\left(\rho_{M 23}^{\prime}, \theta_{M 23}^{\prime}\right) .
$$

To decide if the focus level is satisfactory, the grid similarity is computed through intensity correlation [6], an object function also employed in the aforementioned MD routine. If the normalized correlation sum in (13) is adequately close to unity, the aperture image is autofocused; otherwise, another geometry hypothesis must be assessed.

$$
C=\frac{\sum \sum\left(g_{12}-m_{12}\right) \cdot\left(g_{23}-m_{23}\right)}{\sqrt{\left(\sum \sum\left(g_{12}-m_{12}\right)^{2}\right) \cdot\left(\sum \sum\left(g_{23}-m_{23}\right)^{2}\right)}} .
$$

In (13), $g_{12}=\left|I_{12}\left(\rho_{M 12}^{\prime}, \theta_{M 12}^{\prime}\right)\right|^{2}$ and $g_{23}=\left|I_{23}\left(\rho_{M 23}^{\prime}, \theta_{M 23}^{\prime}\right)\right|^{2}$ are magnitude squared grids; corresponding average values are denoted by $m_{12}$ and $m_{23}$. Note also that the summation in (13) is carried out over all pixels.

An exhaustive 6-D geometry search is not a feasible approach in practice, especially not if several autofocus steps (factorization steps with adjustable geometry parameters) are required, i.e., if the algorithm is activated early on in the processing chain [17]. However, for a near-linear aperture track, the sensitivity is low for $H_{13}, \phi$, and $\beta_{13}$ (external triangle quantities), potentially limiting the FGA algorithm to a variation of $v, \Delta L$, and $L_{13}$ (internal triangle quantities). Naturally, this facilitates the autofocus problem. If the size of the scene is restricted as well, it may even be viable to vary a single parameter, such as $v$. In general though, the preceding premises cannot be taken for granted, as the number of geometrical quantities needed to retrieve a focused image is influenced by wavelength, bandwidth, resolution, IMU/GPS measurement accuracy, scene size, etc. Hence, the strategy must be able to correct (by means of an alternative search routine) six parameters if necessary.

This paper will, however, in contrast, characterize a couple of challenging problems confined to one quantity. Thus, the ability to mitigate residual space variant RCM will be validated in a rather academic fashion. In essence, this paper resumes a first move, demonstrating the capacity of the new algorithm, prior to performing a full trial (left for future work).

\section{E. Data Sets}

The FGA algorithm has been applied on two synthetic and two real data sets.

Synthetic data, i.e., for two different scenes (point targets with and without noise), are generated in stripmap mode along linear aperture tracks by a simulated CARABAS-II-like system. The track length is, however, erroneous (the true track was corrupted before processing to model measurement errors), motivating the use of autofocus (a variation of $L_{13}$; see next section for more details).

Real data, i.e., for two different scenes (Vidsel and Linköping), are acquired in stripmap mode along nonlinear aperture tracks by the CARABAS II system [18]. The track scale is, however, erroneous (the measured track was corrupted before processing to model measurement errors), motivating the use of autofocus (a scale factor, i.e., $s_{f}$, variation or, essentially, a simultaneous alteration of $L_{13}, \Delta L$, and $H_{13}$; see next section for more details).

It should also be stressed that radar echoes from a limited number of sample positions have been factorized and focused (partial tracks processed for both synthetic and real data sets). This, in turn, indicates that the integration angle (and the resolution) varies across the scenes as in spotlight mode.

In the context of data generation/acquisition, common SAR premises, e.g., the start-stop and Born approximations, as well as a constant wave velocity, are also adopted (see [27] for further information).

The first synthetic data set consists of 21 point targets. This represents an ideal scenario.

The second synthetic data set consists of 441 point targets and noise. This scenario provides an opportunity to verify that the new algorithm is robust (in the presence of noise), before testing it on real data. In this case, the signal-to-noise ratio (SNR) was adjusted to approximately $20 \mathrm{~dB}$ (complex white Gaussian noise, target at scene center used as a reference, i.e., target peak power divided by average noise power in the final aperture image). The additional targets, in turn, were added to increase the total signal energy and, thus, the reliability of the correlation.

The first real data set (Vidsel) originates from a rural scene with a sparse forest region and a lake. A few buildings, a trihedral reflector, and a power line structure are the primary targets.

The second real data set (Linköping) originates from an elaborate urban scene, which is swamped with buildings and other man-made objects.

Data-related information (i.e., system and geometry quantities) is resumed in Table I.

\section{F. Realization and Evaluation}

The first realization task is to form sub-images by employing back-projection on adjacent segments (sub-apertures) of a track. Equation (14) is a general back-projection expression, i.e.,

$$
I(\rho, \theta)=\sum_{n=x}^{M+x-1} f(n, R) \cdot R \cdot \exp \left(\frac{ \pm j 4 \pi R}{\lambda_{c}}\right)
$$

where $I$ is the sub-image for a segment extending across $M$ slow time positions, i.e., $n$, starting at position $x$. Radar echoes, i.e., $f(n, R)$, are presumed to be pulse compressed and demodulated. The slant range between the position and the 
TABLE I

Resumed System AND GeOMETRY QuAntities. For Synthetic DATA, THE Size of the Scene Is Rather Restricted to SAVE Run Time AND MEMORY. For CARABAS II DATA, THE Full INTEGRATION Angle $\left(\sim 70^{\circ}\right)$ Is Never Processed; Thus, in That Sense, Real Scenes Are Restricted in Size as Well. Note Also That Images SHOWN FOR CARABAS II DATA ARE CUTS OF IMAGES FORMED FOR The SCEne Size in the Table below. The SAmple SPacing (AzImuth AND Slant RANGE) Is, IN TURN, REPORTED FOR Image Data, Not Raw Radar Data. The SQuint ANGLE $\left(0^{\circ}\right)$ FinALLY IMPLIES BROADSIDE IMAGING

\begin{tabular}{lrr}
\hline Quantity & Syn. data set 1/2 & CII data set 1/2 \\
\hline SAR mode & Stripmap & Stripmap \\
Centre frequency & $55 \mathrm{MHz}$ & $55 \mathrm{MHz}$ \\
Bandwidth & $70 \mathrm{MHz}$ & $70 \mathrm{MHz}$ \\
Range spacing & $0.83 \mathrm{~m}$ & $0.47 \mathrm{~m}$ \\
Azimuth spacing & $0.98 \mathrm{~m}$ & $0.97 \mathrm{~m} / 1.09 \mathrm{~m}$ \\
Integration angle & $\sim 45^{\circ}-75^{\circ}$ & $\sim 45^{\circ}-60^{\circ}$ \\
Squint angle & 0 & $0^{\circ}$ \\
Range (scene centre) & $1800 \mathrm{~m}$ & $\sim 16000 \mathrm{~m}$ \\
Size of scene & $1000 \times 1000 \mathrm{~m}$ & $\sim 15400 \times 6100 \mathrm{~m}$ \\
Aperture length & $2000 \mathrm{~m}$ & $\sim 15400 \mathrm{~m}$ \\
Altitude & $750 \mathrm{~m}$ & $6400 \mathrm{~m} / 4500 \mathrm{~m}$ \\
Number of targets & $21 / 441$ & $\mathrm{~N} / \mathrm{A}$ \\
\hline
\end{tabular}

pixel coordinate (i.e., $\rho$ and $\theta$ ) in question is denoted by $R$; the range multiplication is included to establish $1 / R$ dependence (see [27] for further information), whereas the exponential is a phase factor (demodulated data) with center wavelength, i.e., $\lambda_{c}$ (i.e., the wavelength at the center frequency of the system).

Complex $f(n, R)$ values are found through nearest neighbor interpolation of discrete (in range as well) radar echoes $f\left(n, R_{d}\right)$, upsampled eight times in slant range (giving a sample spacing about 16 times finer than Nyquist for synthetic data, i.e., due to an initial oversampling factor; for real data, a sample spacing about 32 times finer than Nyquist is attained, i.e., due to an initial upsampling factor) through zero padding in the (range) frequency domain.

For synthetic data sets, 16 sub-images are produced (four factorization steps feasible). For real data sets, eight sub-images are produced (three factorization steps feasible). Sub-apertures are factorized (with 2-D cubic spline interpolation) until a (final) reference aperture image is attained. A polar to Cartesian conversion is then carried out (with 2-D cubic spline interpolation), giving an azimuth and slant range representation, contrary to a polar representation in the $x y$-plane. After applying a ramp filter in the 2-D frequency domain (to even out the spectrum; see [27] for further information), resolution (3 dB in azimuth and slant range) and peak-to-sidelobe ratio (PSLR) are measured for a few targets in the (reference) image. In the case of synthetic data, values are compared with theory [28], [29] and confirmed to agree well. Erroneous tracks, causing residual space variant RCM, are then specified according to the following principle.

Denote the slant range between the center of a linear aperture track at constant altitude and a broadside (at the center) point target by $R_{b}$. The length of the aperture is, in turn, denoted by $l$. Presume a symmetrical error and denote the erroneous aperture length by $l_{e}$. Target range is measured along both apertures; if the absolute range difference, i.e., $|\Delta R|$, exceeds the width of
TABLE II

RESUMED ERROR QUANTITIES FOR THE TRACKS $\left(\delta_{R} \approx 2.1 \mathrm{~m} ; \delta_{R}\right.$ VARIES ACROSS THE SCENE [28], [29]). NOTE THAT THE MAGNitUde OF the SPaCe-Variant EFFects Is the SAme For Synthetic and Real

Data Sets. The Residual RCM (CAlculated at Near Range) IS MORE DISTINCT FOR CARABAS II DATA

\begin{tabular}{lrr}
\hline Quantity & Syn. data set 1/2 & CII data set 1/2 \\
\hline$l$ & $2000 \mathrm{~m}$ & $\sim 15400 \mathrm{~m}$ \\
$l_{e}$ & $2050 \mathrm{~m}$ & $\sim 15477 \mathrm{~m}$ \\
$\Delta R$ & $\sim 7.3 \delta_{R}$ & $\sim 9.4 \delta_{R}$ \\
$\Delta R_{n}-\Delta \mathrm{R}_{\mathrm{f}}$ & $\sim 2.5 \delta_{R}$ & $\sim 2.5 \delta_{R}$ \\
$s_{f}$ & 1.025 & 1.005 \\
\hline
\end{tabular}

half a (range) resolution cell, i.e., $\delta_{R}$, RCM is introduced, with the criterion

$$
|\Delta R|=\left|\left(\sqrt{\left(R_{b}^{2}+\frac{l_{e}^{2}}{4}\right)}-\sqrt{\left(R_{b}^{2}+\frac{l^{2}}{4}\right)}\right)\right|>\frac{\delta_{R}}{2} .
$$

If the absolute difference in $\Delta R$ for targets with differing $R_{b}$ ( $R_{b n}$ and $R_{b f}$, for example, representing near and far ranges, respectively) exceeds the width of half a resolution cell, space variant effects are introduced as well, with the criterion

$$
\begin{array}{r}
\left|\Delta R_{n}-\Delta R_{f}\right|=\mid\left(\sqrt{\left(R_{b n}^{2}+\frac{l_{e}^{2}}{4}\right)}-\sqrt{\left(R_{b n}^{2}+\frac{l^{2}}{4}\right)}\right. \\
\left.-\sqrt{\left(R_{b f}^{2}+\frac{l_{e}^{2}}{4}\right)}+\sqrt{\left(R_{b f}^{2}+\frac{l^{2}}{4}\right)}\right) \mid>\frac{\delta_{R}}{2} .
\end{array}
$$

Equations (15) and (16) fit the synthetic data model, assuming a linear aperture track and an erroneous aperture length. For real data, only along-track terms are accounted for. This is a valid approximation for minor cross-track and altitude alterations (experimentally confirmed for CARABAS II data). Equation (17) converts the length error to a suitable faulty scale factor $s_{f}$, i.e.,

$$
s_{f}=\frac{l_{e}}{l} .
$$

False track parameters satisfying the criteria in (15) and (16) are now introduced before the back-projection. Criteria-related information (i.e., error quantities for the tracks) is resumed in Table II. Resulting sub-images are still focused. The following principle assures this.

Denote the slant range between the center of a linear subaperture track at constant altitude and a broadside (at the center) point target by $R_{b}$. The length of the sub-aperture is denoted by $l_{s}$, whereas the along-track position is symbolized by $x_{s}\left(x_{s}=\right.$ 0 at the sub-aperture center; positions are assumed equidistant). Presume a symmetrical error and denote the erroneous subaperture length by $l_{\mathrm{se}}$; the erroneous along-track position is symbolized by $x_{\mathrm{se}}\left(x_{\mathrm{se}}=s_{f} \cdot x_{s}\right)$; note that the expression within the parenthesis assumes that the ratio between $l_{\mathrm{se}}$ and $l_{s}$ is the same as that for the full aperture [see (17)]. Target range is measured (as a function of $x_{s}$ ) along both sub-apertures, and the range difference, i.e., $\Delta R_{s}\left(x_{s}\right)$, is derived

$$
\Delta R_{s}\left(x_{s}\right)=\sqrt{\left(s_{f}^{2} \cdot x_{s}^{2}+R_{b}^{2}\right)}-\sqrt{\left(x_{s}^{2}+R_{b}^{2}\right)} .
$$




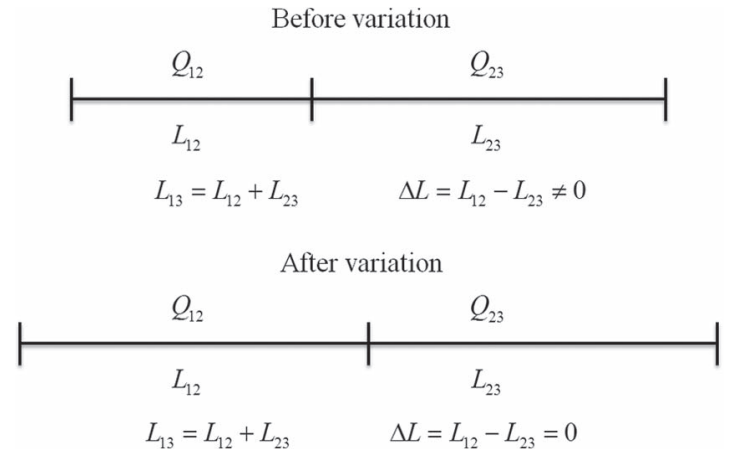

Fig. 4. (Top) Example where the previous geometry variation has given two different $L_{13}$ values, i.e., differing $L_{12}$ and $L_{23}$ values prior to the present variation. (Bottom) After varying the geometry, a new $L_{13}$ value is adopted under the premise that $\Delta L$ is equal to zero.

Equation (18) is Taylor expanded around $x_{s}=0$; secondorder coefficients are retained. Thus

$$
\Delta R_{s q}\left(x_{s}\right)=\frac{\left(s_{f}^{2}-1\right)}{R_{b}} \cdot \frac{x_{s}^{2}}{2} .
$$

Peak-to-center magnitudes of the quadratic expression in (19) should be held below $\lambda_{c} / 16$ [6], [20] to keep sub-images focused, with the criterion

$$
\left|\Delta R_{s q}\left(l_{s} / 2\right)\right|=\frac{\left|\left(s_{f}^{2}-1\right)\right|}{R_{b}} \cdot \frac{l_{s}^{2}}{8}<\frac{\lambda_{c}}{16} .
$$

For real data, once again, only along-track terms are accounted for. This approximation has also been experimentally confirmed.

For synthetic data, magnitudes in (20) are verified to be well below $\lambda_{c} / 16$ ( $\sim 6$ times lower). For real data, the magnitudes are very close to $\lambda_{c} / 16$; at near range, the focus criterion is actually exceeded by about $\lambda_{c} / 300$. This is a deliberate choice, to really challenge the novel strategy.

Sub-apertures are factorized with the FGA algorithm.

For synthetic data, $L_{13}$ is varied step by step (i.e., four autofocus steps, FGA starting with 16 sub-images/sub-apertures), whereas $\Delta L$ is set to zero regardless of prior hypotheses (see Fig. 4). By following this approximate approach, the autofocus problem is constrained to one geometrical parameter.

For real data, $s_{f}$ is varied step by step (i.e., three autofocus steps, FGA starting with eight sub-images/sub-apertures). Before proceeding to the next autofocus step, an average scale factor is calculated and employed when forming (definite) new sub-images (see Fig. 5). This approximate approach preserves all angles, and the autofocus problem is once again constrained to one geometrical parameter.

Note that the FGA algorithm ideally should converge to the correct $L_{13} / s_{f}$ value on the first step. However, due to noise, numerical issues, etc., this is not expected in practice.

After the (final) autofocused aperture image has been attained, converted to Cartesian form, and ramp filtered, resolution ( $3 \mathrm{~dB}$ in azimuth and slant range) and PSLR are measured for the same targets as in the reference image. Apart from focused (reference) and autofocused (FGA) images, defocused (faulty sub-apertures factorized without autofocus) and PGA-

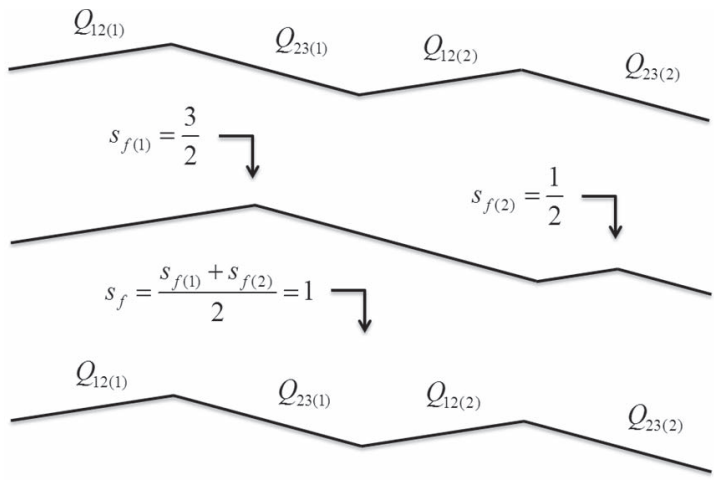

Fig. 5. Example where scales for two pair of sub-apertures have been individually varied. The best focus measures have been found for different factors. However, before actually forming (definite) new sub-images (proceeding to the next autofocus step), the factors are averaged, and the mean is used to scale the pairs as a unit.

processed images [20] are also obtained. The complete collection enables a comprehensive evaluation.

It should be mentioned that, for synthetic data with noise, the preceding recap must be modified a bit, to regard that the noise is removed before performing target measurements and presenting images. Fundamentally, the FGA algorithm (and the PGA) is first applied on data with noise. The corresponding autofocus solution is then used to process data without noise (i.e., the noise is excluded when generating data), giving the FGA (and the PGA) image. The reference and the defocused image can be formed right off, i.e., after excluding the noise.

\section{RESULTS}

Results for both synthetic and real data sets will now be presented, figure by figure.

The first synthetic data set (see Figs. 6-9) covers a $1 \mathrm{~km}^{2}$ area (without noise). The erroneous aperture length (2050 m instead of $2000 \mathrm{~m}$ ) causes residual space variant RCM; this can be theoretically confirmed by means of (15), (16), and the information in Table I. The defocused image is corrected (true aperture length found within $\pm 5 \mathrm{~cm}$ ) after two out of four autofocus steps. Measurements on all 21 targets in the FGA image reveal that resolution (azimuth and slant range) and PSLR are very close to corresponding reference values, i.e., within $\pm 1.0 \%$ and $\pm 0.05 \mathrm{~dB}$. Note that, before measuring, target chips are extracted and upsampled 50 times by way of zero padding in the 2-D frequency domain.

The second synthetic data set (see Figs. 10-13) covers the same $1 \mathrm{~km}^{2}$ area (with noise) as before. The erroneous aperture length ( $2050 \mathrm{~m}$ instead of $2000 \mathrm{~m}$ ) is, in this case, corrected (true aperture length found within $\pm 5 \mathrm{~cm}$ ) after four out of four autofocus steps. Measurements on 21 targets (out of 441, same point targets and upsampling factor as before) in the FGA image reveal that resolution (azimuth and slant range) and PSLR are very close to corresponding reference values, i.e., within $\pm 1.0 \%$ and $\pm 0.10 \mathrm{~dB}$.

The first real data set, i.e., Vidsel (see Figs. 14-18), covers an $\sim 3.5 \mathrm{~km}^{2}$ area (maximal area shown; see Table I). The erroneous scale factor (1.0050 instead of 1.0000) causes residual 

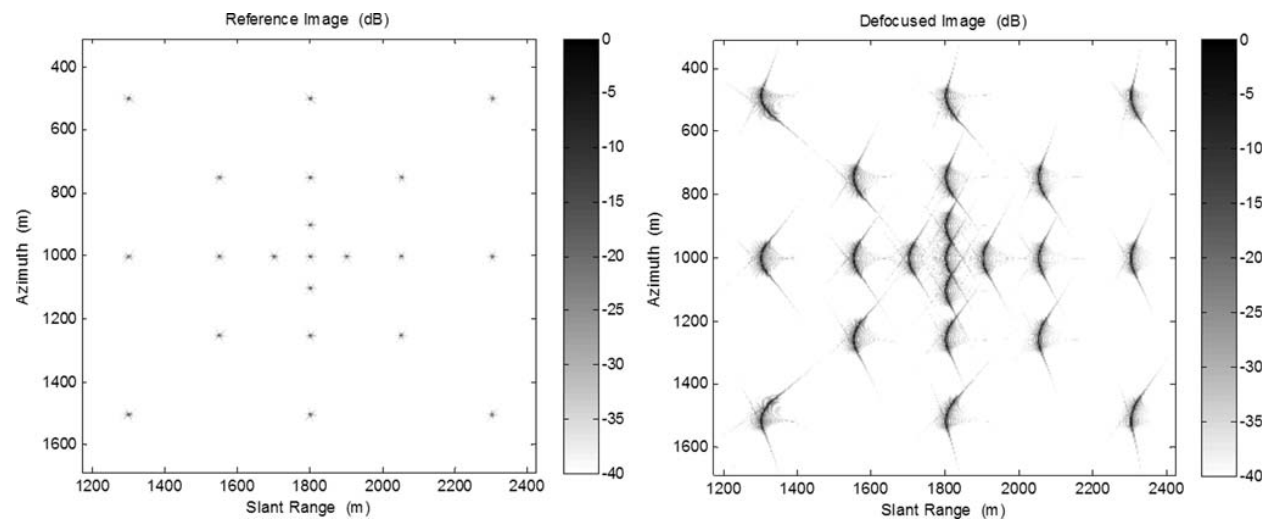

Fig. 6. (Left) Reference and (right) defocused images for the first synthetic data set. The defocused image displays residual space variant RCM (i.e., curved target trajectories are not shaped the same).
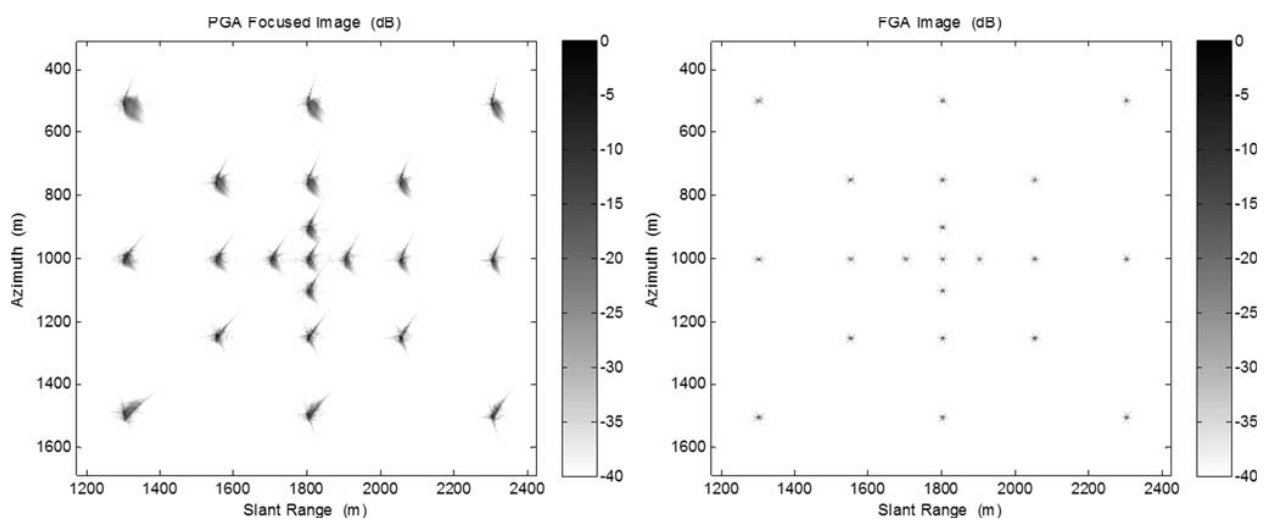

Fig. 7. (Left) PGA-processed and (right) FGA images. The PGA result is far from satisfying; residual space variant RCM is still seen. The FGA image is visually very similar (essentially identical) to the reference image in Fig. 6.
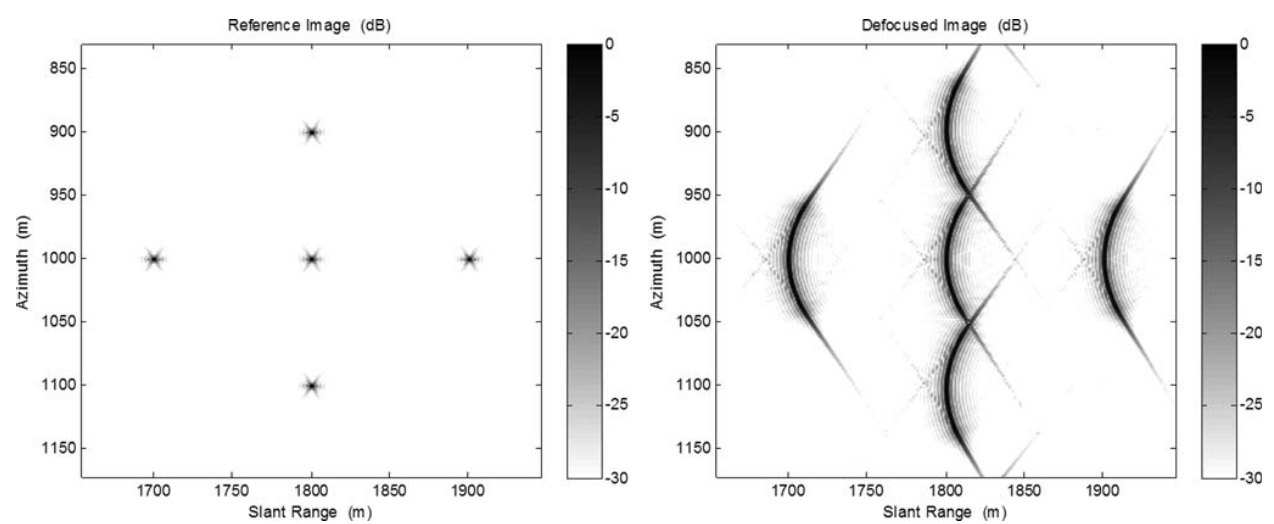

Fig. 8. Close-up view of five central targets in Fig. 6. (Left) In the reference image, targets are compressed with crossed sidelobes, a typical UWB attribute. (Right) In the defocused image, vertically aligned trajectories link up.
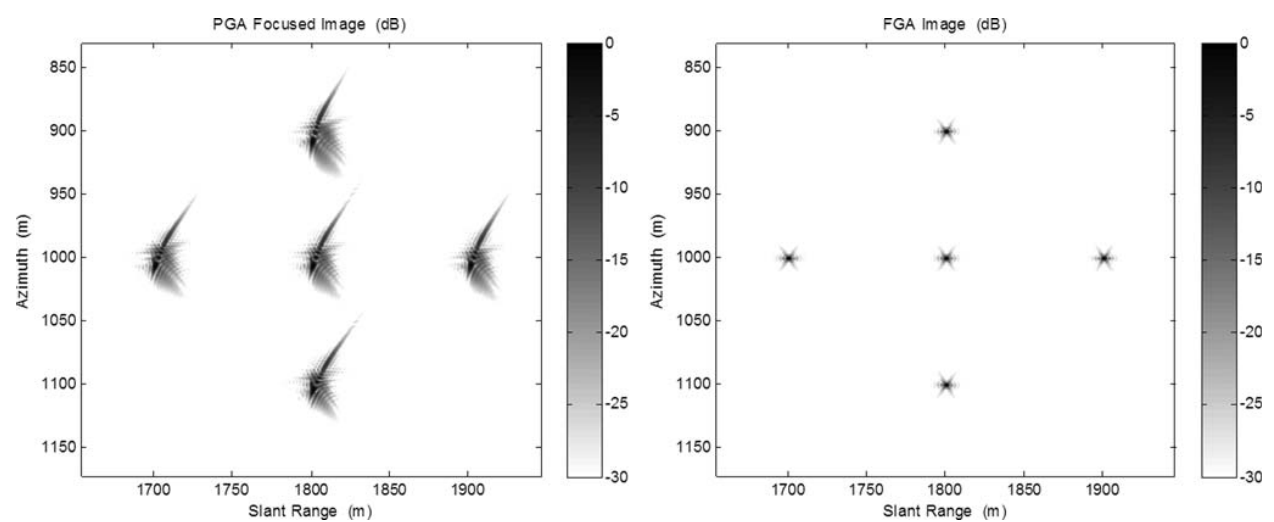

Fig. 9. Close-up view of five central targets in Fig. 7. It is evident that the performance of the PGA is reduced in this case, as opposed to the geometrical (FGA) approach. 

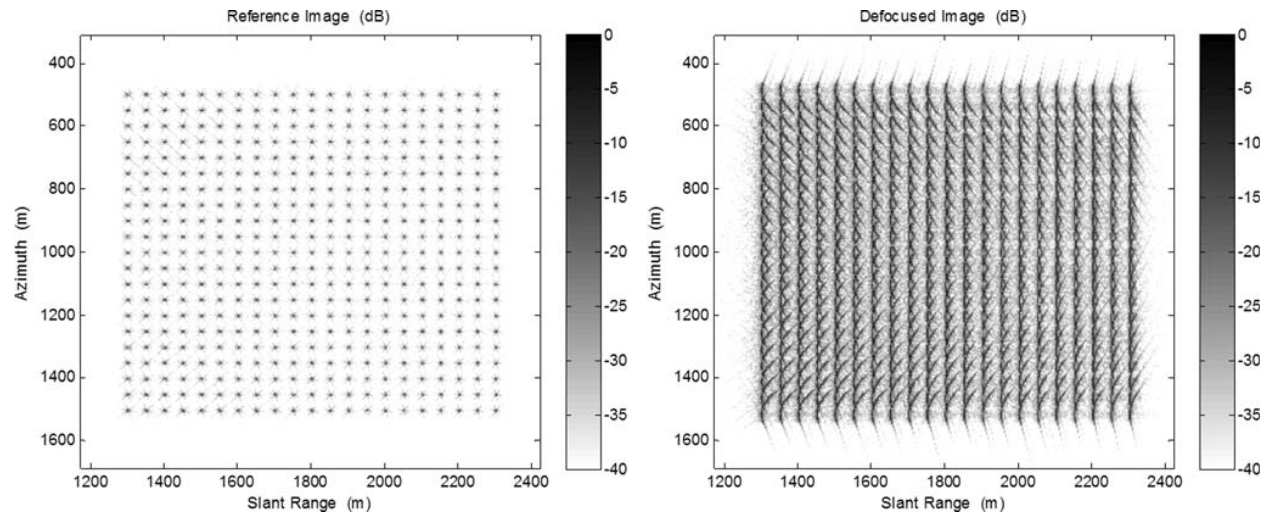

Fig. 10. (Left) Reference and (right) defocused images for the second synthetic data set. The reference image has an ordered structure (targets placed $50 \mathrm{~m}$ apart), whereas the defocused image is very chaotic as trajectories interfere.
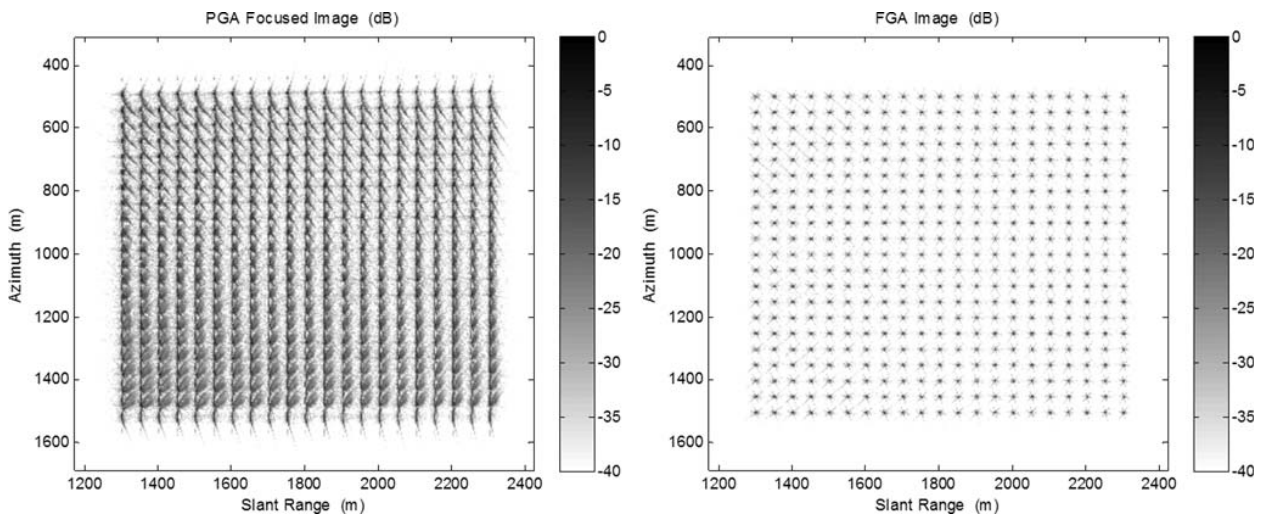

Fig. 11. (Left) PGA-processed and (right) FGA images. The PGA result is once again far from satisfying. The FGA image is visually very similar (essentially identical) to the reference image in Fig. 10.
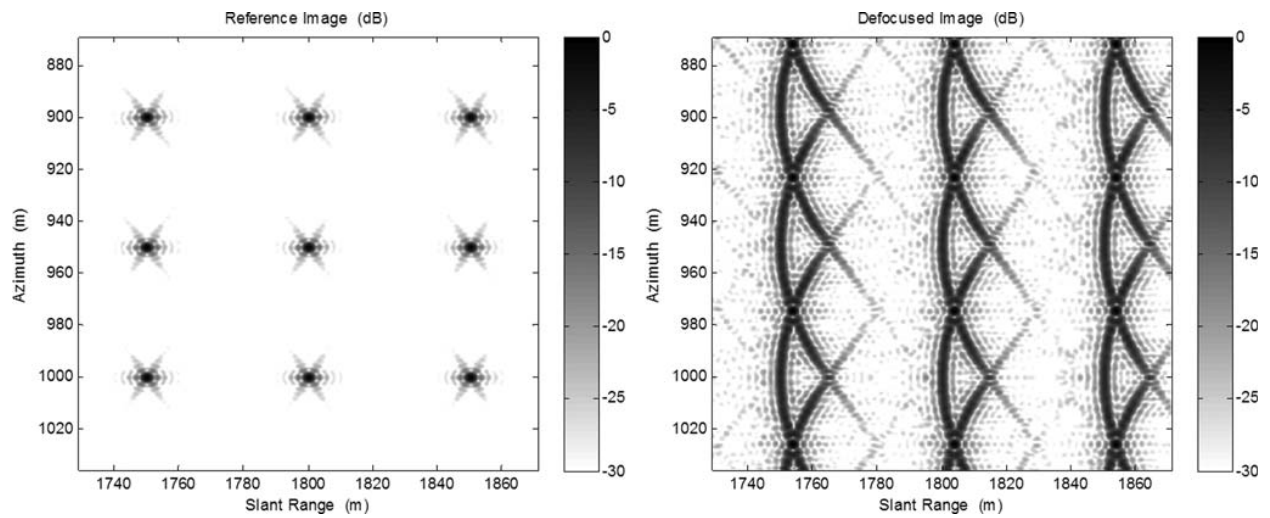

Fig. 12. Close-up view of nine central targets in Fig. 10. (Left) In the reference image, targets are compressed with crossed sidelobes, a typical UWB attribute. (Right) In the defocused image, vertically aligned trajectories overlap.
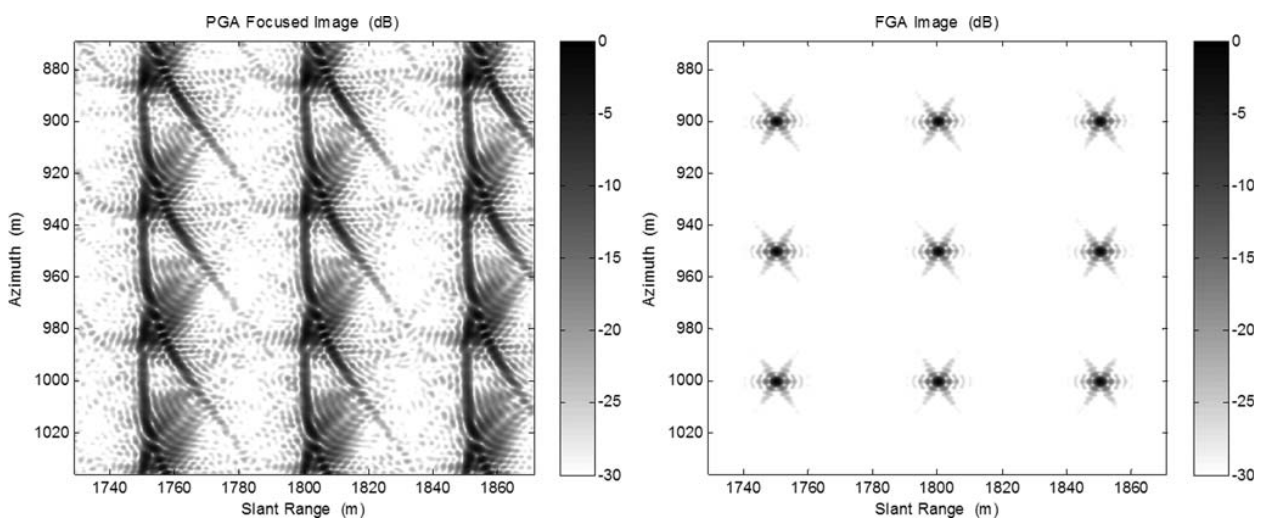

Fig. 13. Close-up view of nine central targets in Fig. 11. It is once again evident that the performance of the PGA is reduced, as opposed to the geometrical (FGA) approach. 

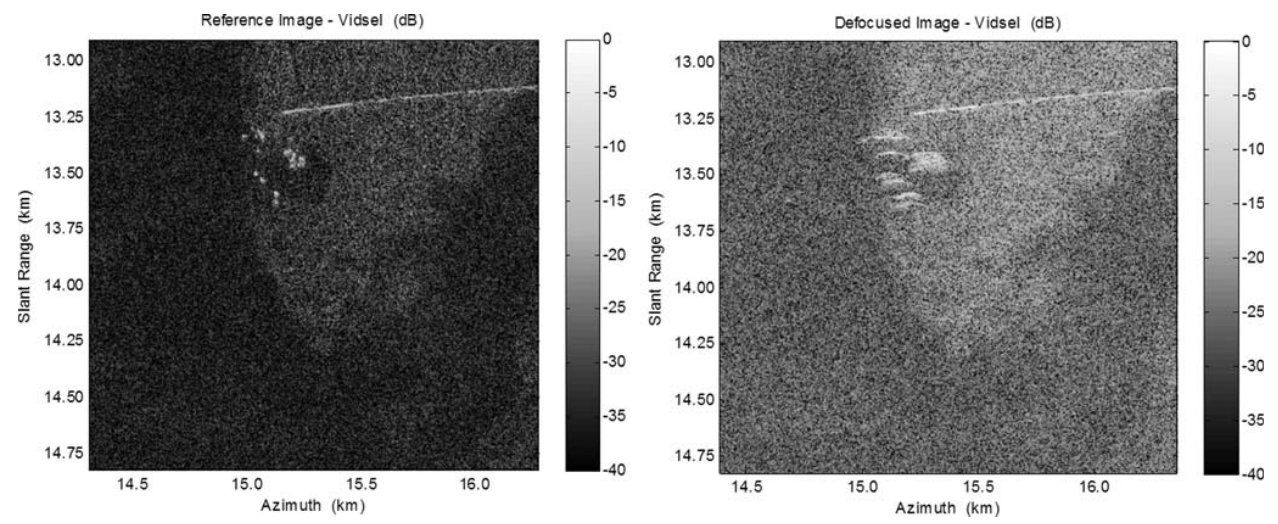

Fig. 14. (Left) Reference and (right) defocused images for the first real data set (CARABAS II—Vidsel). The defocused image displays residual space invariant RCM.
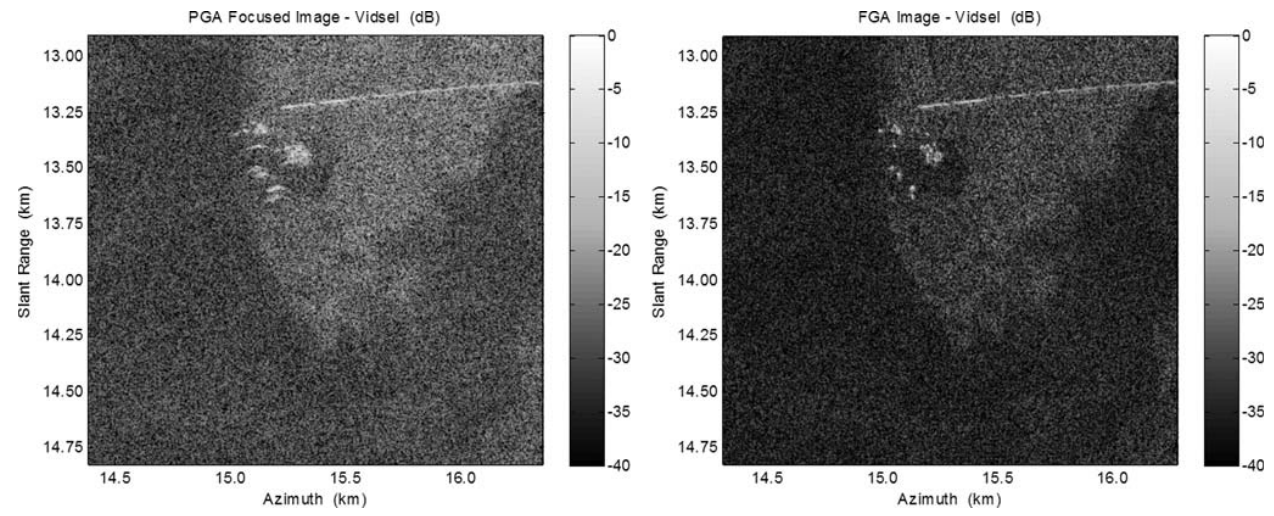

Fig. 15. (Left) PGA-processed and (right) FGA images. The PGA result is not satisfying; residual space invariant RCM is still seen. The FGA image is visually very similar to the reference image in Fig. 14.
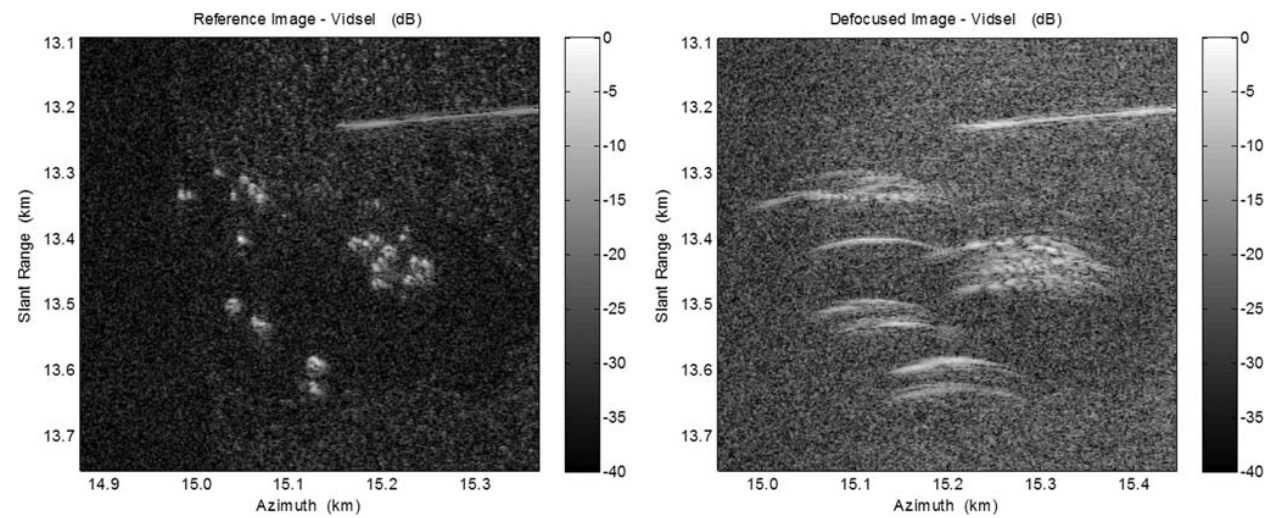

Fig. 16. Close-up view of Fig. 14. The SNR degradation in the defocused image is obvious; the residual RCM is very observable as well.
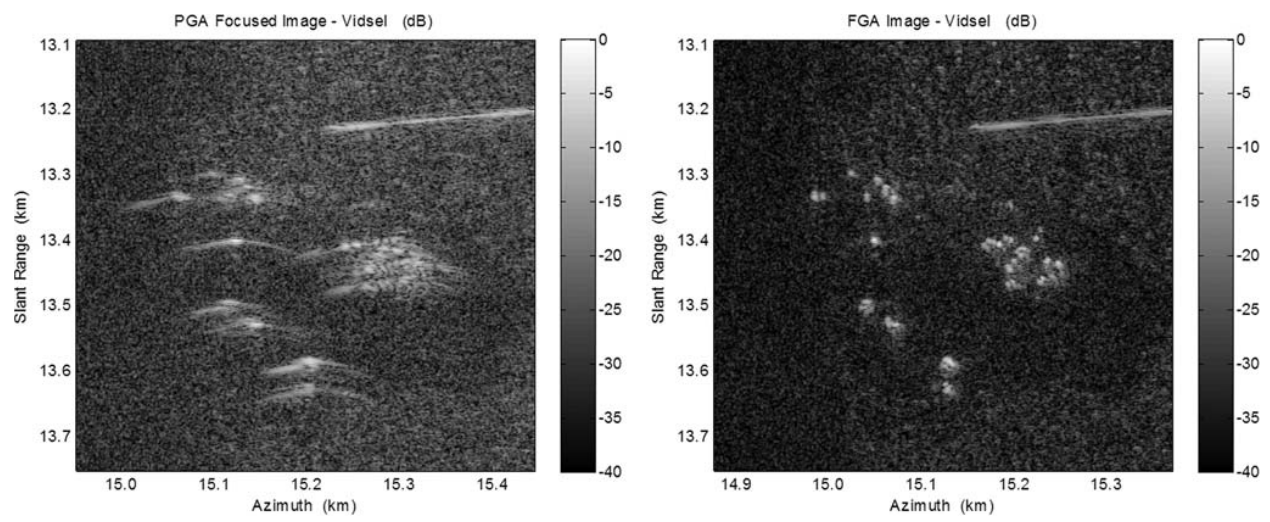

Fig. 17. Close-up view of Fig. 15. The FGA result is promising; the PGA result, on the other hand, is not acceptable. 

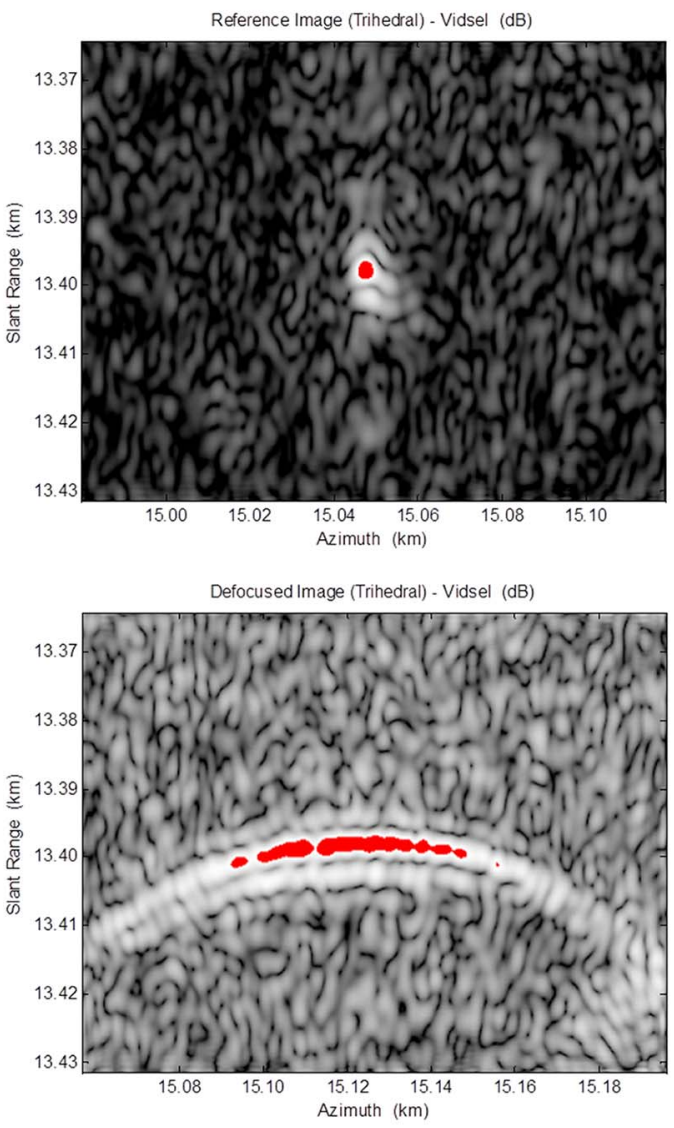

PGA Focused Image (Trihedral) - Vidsel (dB)
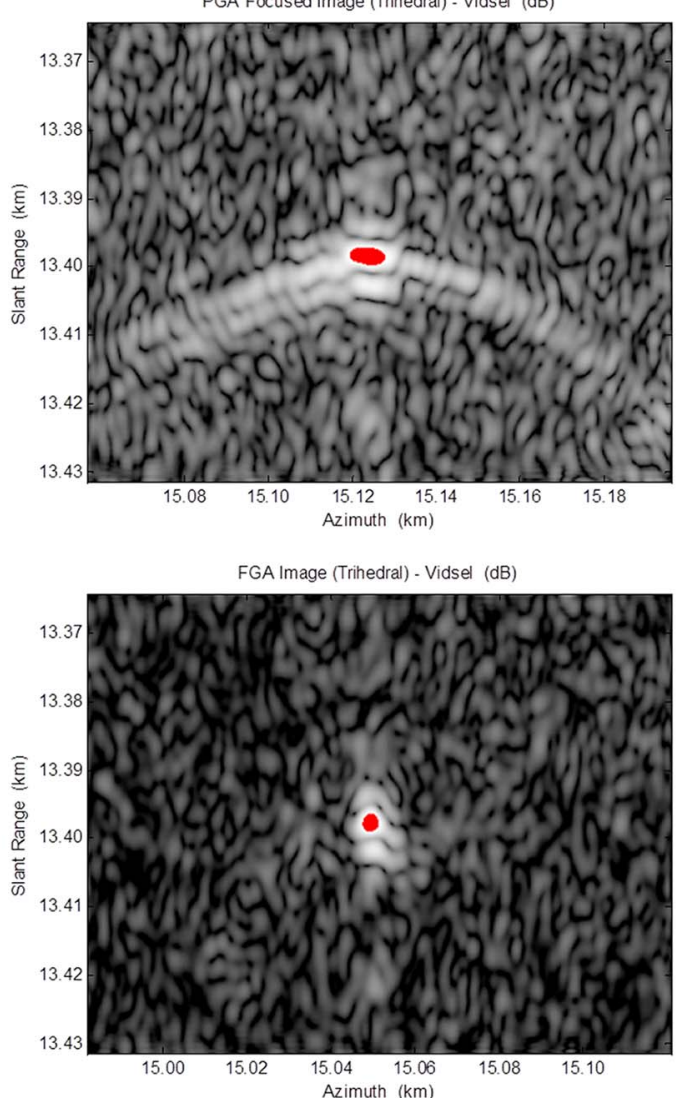

Reference Mesh Plot (Trihedral) - Vidsel (Normalized Magnitude)

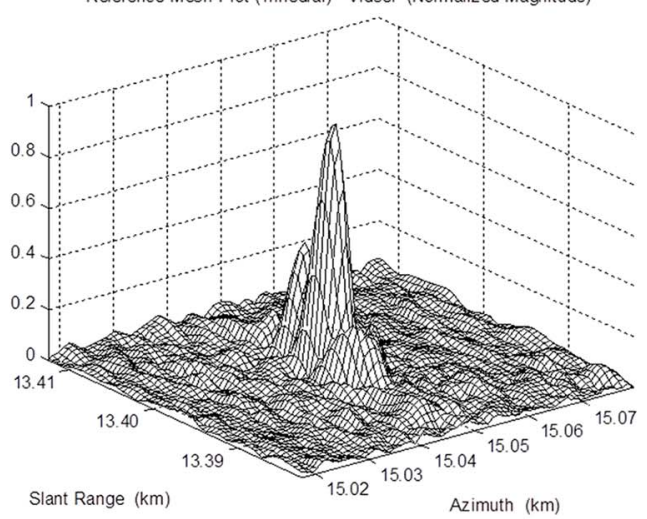

Defocused Mesh Plot (Trihedral) - Vidsel (Normalized Magnitude)

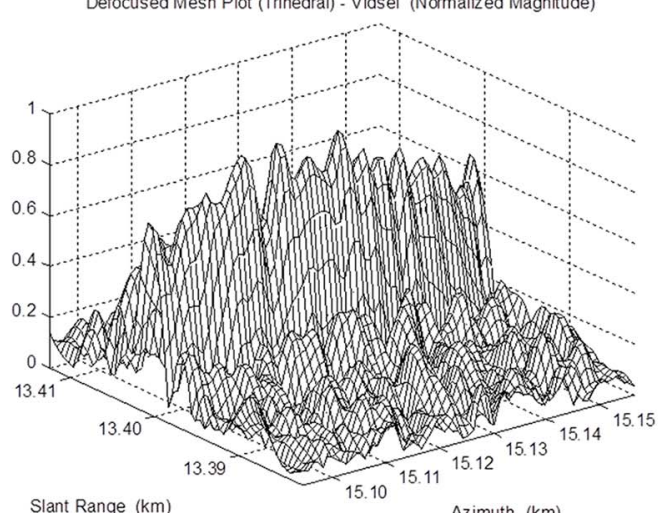

PGA Focused Mesh Plot (Trihedral) - Vidsel (Normalized Magnitude)

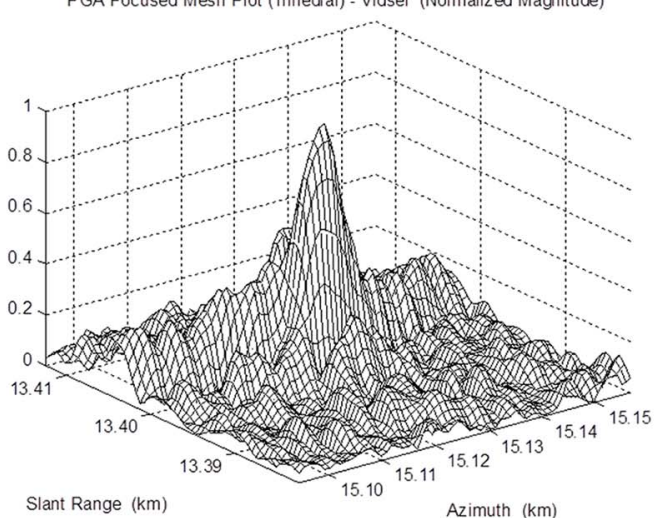

FGA Mesh Plot (Trihedral) - Vidsel (Normalized Magnitude)

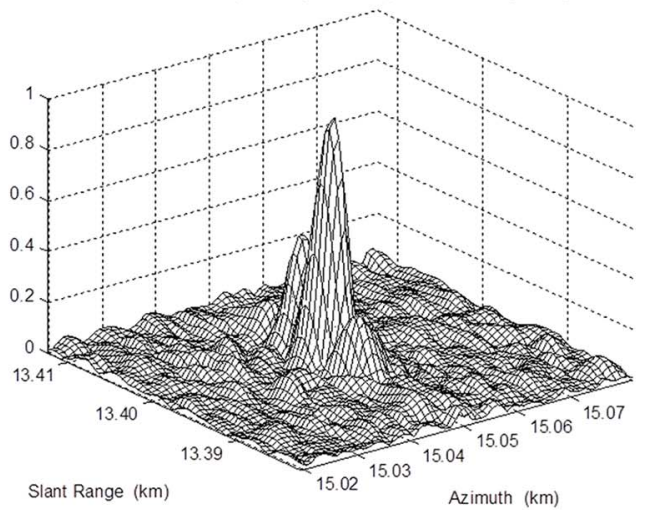

Fig. 18. (Left, top to bottom) Image chips for the trihedral; 3-dB areas are marked red. (Right, top to bottom) Three-dimensional mesh plots. Note in particular that the mainlobe in the PGA image is blurred, with a weak (below the 3-dB level) RCM trace, i.e., although the PGA refines the defocused result, the compression is incomplete. 

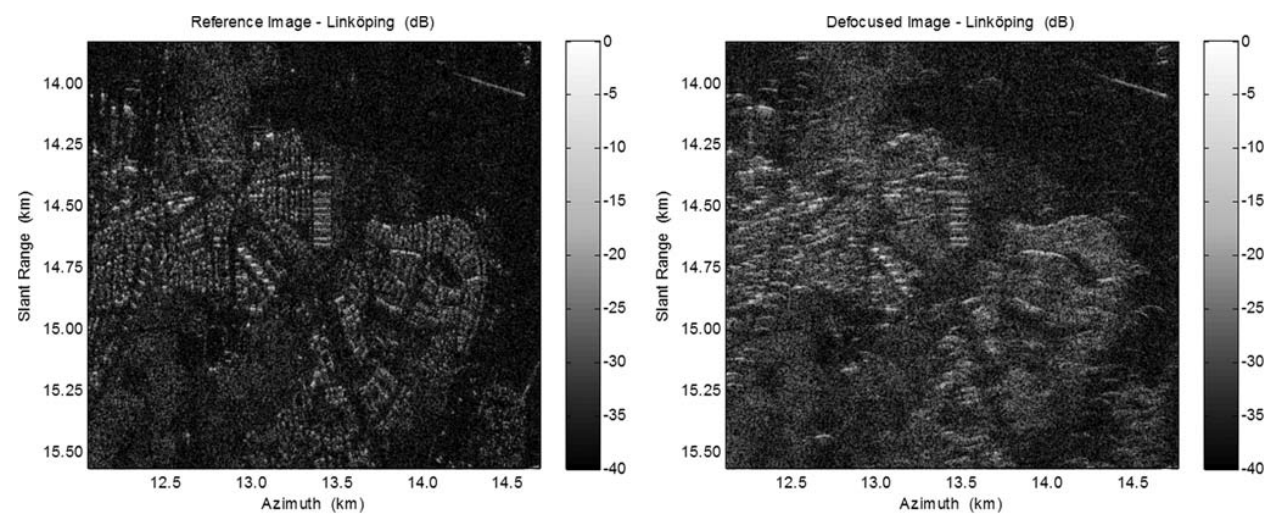

Fig. 19. (Left) Reference and (right) defocused images for the second real data set (CARABAS II—Linköping). The defocused image displays residual space variant RCM
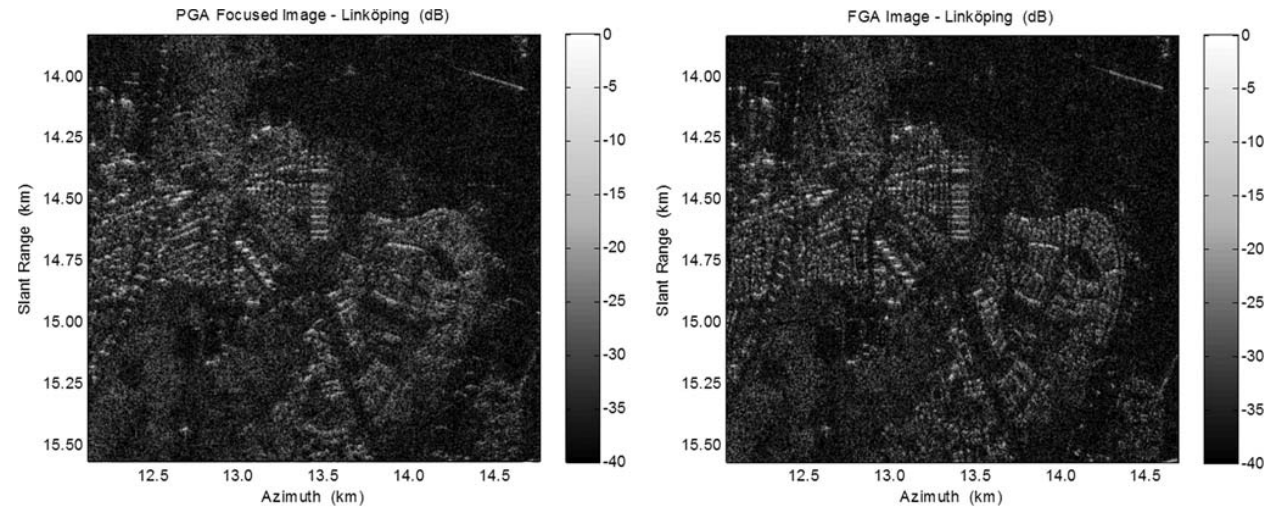

Fig. 20. (Left) PGA-processed and (right) FGA images. The PGA result is not satisfying; residual space variant RCM is still seen. The FGA image is visually very similar to the reference image in Fig. 19.
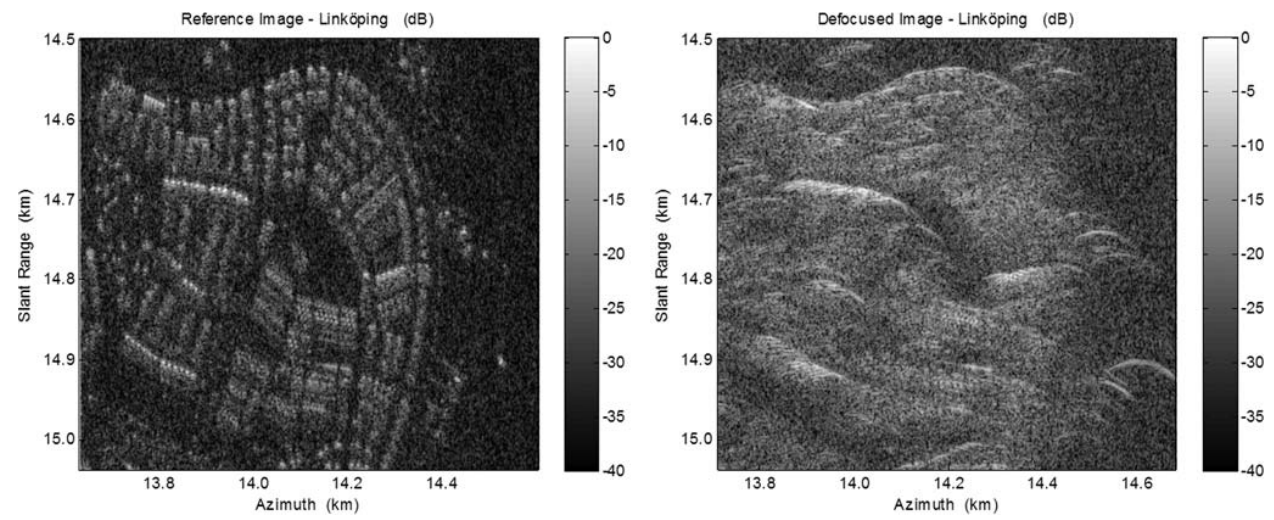

Fig. 21. Close-up view of Fig. 19. The SNR degradation in the defocused image is obvious; the residual RCM is very observable as well.
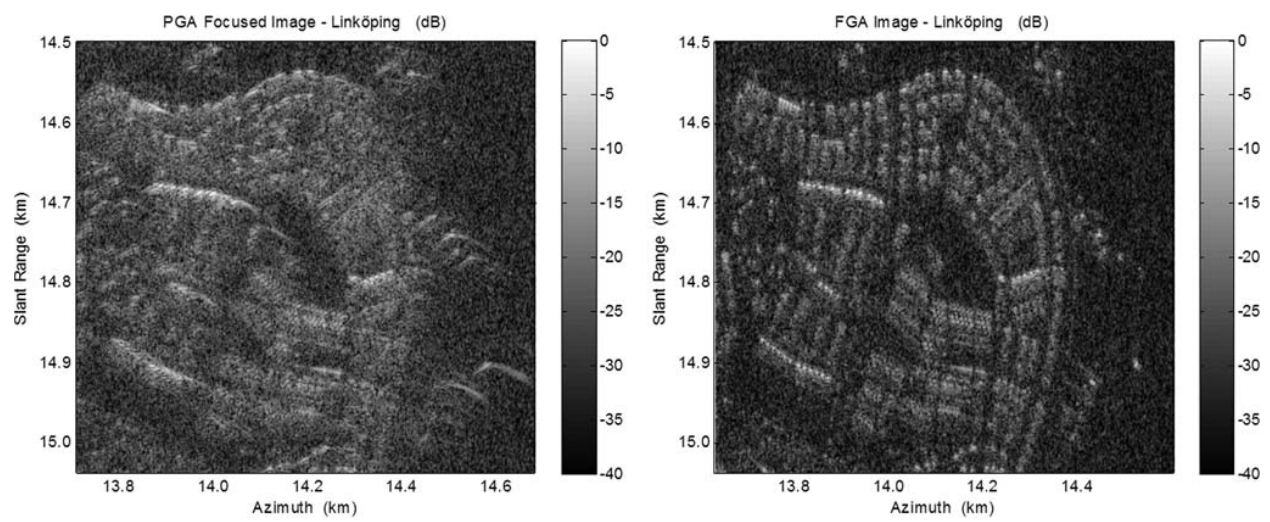

Fig. 22. Close-up view of Fig. 20. The FGA result is promising; the PGA result, on the other hand, is not acceptable. 
space invariant RCM; this can also be theoretically confirmed by means of (15) and the information in Table I. As targets are confined to a small area, i.e., substantially smaller than $3.5 \mathrm{~km}^{2}$, space variant effects according to the criterion in (16) are not seen. The scale factor is estimated to $\sim 1.0049$ after one autofocus step; remaining steps do not correct the scale additionally. Measurements (after upsampling 25 times) on two pointlike targets (including the trihedral reflector) in the FGA image reveal that mainlobes have been broadened by $\sim 2 \%$ in azimuth (3.36-3.44 $\mathrm{m}$ for the reflector) and $\sim 1 \%$ in slant range $(2.38-2.40 \mathrm{~m}$ for the reflector). A PSLR loss of approximately $0.2 \mathrm{~dB}(6.8-6.6 \mathrm{~dB}$ for the reflector) is also observed. Despite the minor degradation (measured), the FGA image is very similar to the reference image. Even when the trihedral is shown alone (see Fig. 18), in form of image cuts and 3-D plots, it is hard to discern a difference.

The second real data set, i.e., Linköping (see Figs. 19-22), covers an $\sim 4.5 \mathrm{~km}^{2}$ area (maximal area shown; see Table I). The erroneous scale factor (1.0050 instead of 1.0000) causes residual space variant RCM; this can also be theoretically confirmed by means of (15), (16), and the information in Table I. The scale factor is estimated to $\sim 1.0050$ after three autofocus steps. Measurements (after upsampling 25 times) on two pointlike targets [located $\sim 1.5 \mathrm{~km}$ apart in slant range, satisfying the criterion in (16)] in the FGA image reveal that the resolution is preserved (no mainlobe broadening). A PSLR loss of approximately $0.2 \mathrm{~dB}$ is observed for the far range target.

PGA-processed images [20] (for both synthetic and real data sets) are not pleasing; this is visually evident; hence, resolution and PSLR have not been measured.

\section{DISCUSSION}

\section{A. Results and FGA}

Results for synthetic and real data sets have now been presented (see Figs. 6-22). It is obvious that focused (reference) and autofocused (FGA) images are very similar. Target measurements also confirm that the FGA algorithm can compensate for residual space variant RCM. A visual inspection verifies that the PGA cannot. This is no surprise since the PGA is a standalone technique (i.e., a separate stage after SAR processing) neglecting the geometrical aspect. By adding an additional autofocus stage or stages within the processing chain [8], [34], [35], it is possible to mitigate residual space invariant RCM. The hybrid approach takes the geometry into consideration, but not completely. The FGA algorithm, on the other hand, is not integrated separately. In fact, as soon as the strategy is activated, it is the processing chain, offering a complete geometrical solution (in this paper though, the problems are confined to one geometrical quantity for simplicity).

Apart from the FGA algorithm (and the early formulation [16]) and the 1-D technique described in [2], back-projection adapted autofocus has been disregarded. This paper, however, promotes the advantages, and although the problems dealt with are constrained, the capacity of the novel strategy is demonstrated, encouraging future work.

\section{B. Future Work}

At the moment, the run time is the main obstacle. The exhaustive search routine must be replaced by a faster alternative, as it makes the FGA algorithm too slow to be of practical use for real-time applications. Gradient-descent-based schemes should be surveyed prior to performing a full trial (i.e., correcting six parameters).

The object function is also an important subject. Although intensity correlation has worked well thus far, there are numerous other functions to consider, e.g., contrast [21], [22], squared intensity [12], entropy [33], etc. [note that these functions are applied on the sum in (12) and not on the addends].

\section{CONCLUSiON}

We have described and analyzed a new geometrical autofocus approach for SAR. The strategy, termed the FGA algorithm, is an FFBP realization with a number of adjustable geometry parameters for each factorization step. By altering the aperture track in the time domain, it is possible to correct an inaccurate geometry (potentially introduced due to relaxed requirements on the IMU/GPS and/or a jammed/shadowed GPS). This indicates that the FGA algorithm has the capacity to compensate for residual space variant RCM.

The performance of the algorithm is demonstrated for geometrically constrained autofocus problems, embracing both synthetic and real (CARABAS II) data sets. Resolution ( $3 \mathrm{~dB}$ in azimuth and slant range) and PSLR measurements on targets (point targets for synthetic data and pointlike targets for real data) in FGA and reference images give similar results within a few percent and tenths of a decibel. The advantage of a geometrical autofocus approach is clarified further when comparing FGA and PGA-processed images [20].

\section{ACKNOWLEDGMENT}

The authors would like to thank Dr. A. Åhlander, J. Lindgren (SAAB Electronic Defence Systems), and Dr. L. Eriksson (Chalmers University of Technology) for their valuable inputs. The authors would also like to thank the anonymous reviewers for their comments, helping to improve this paper.

\section{REFERENCES}

[1] L.-E. Andersson, "On the determination of a function from spherical averages," Siam J. Math. Anal., vol. 19, no. 1, pp. 214-232, Jan. 1988.

[2] J. N. Ash, "An autofocus method for backprojection imagery in synthetic aperture radar," IEEE Geosci. Remote Sens. Lett., vol. 9, no. 1, pp. 104 108, Jan. 2012.

[3] C. Cafforio, C. Prati, and F. Rocca, "SAR data focusing using seismic migration techniques," IEEE Trans. Aerosp. Electron. Syst., vol. 27, no. 2, pp. 194-207, Mar. 1991.

[4] H. Cantalloube and P. Dubois-Fernandez, "Airborne X-band SAR imaging with $10 \mathrm{~cm}$ resolution: Technical challenge and preliminary results," Proc. Inst. Elect. Eng.-Radar, Sonar Navigat., vol. 153, no. 2, pp. 163-176, Apr. 2006.

[5] H. M. J. Cantalloube and C. E. Nahum, "Multiscale local map-drift-driven multilateration SAR autofocus using fast polar format image synthesis," IEEE Trans. Geosci. Remote Sens., vol. 49, no. 10, pp. 3730-3736, Oct. 2011.

[6] W. G. Carrara, R. S. Goodman, and R. M. Majewski, Spotlight Synthetic Aperture Radar: Signal Processing Algorithms. Norwood, MA, USA: Artech House, 1995. 
[7] H. L. Chan and T. S. Yeo, "Noniterative quality phase gradient autofocus (QPGA) algorithm for spotlight SAR imagery," IEEE Trans. Geosci. Remote Sens., vol. 36, no. 5, pp. 1531-1539, Sep. 1998.

[8] A. W. Doerry, "Autofocus correction of SAR images exhibiting excessive residual migration," in Proc. SPIE Radar Sensor Technol. IX, 2005, vol. 5788, pp. 34-35.

[9] P. H. Eichel, D. C. Ghiglia, and C. V. Jakowatz, "Speckle processing method for synthetic-aperture-radar phase correction," Opt. Lett., vol. 14, no. 20 , pp. $1-3,1989$.

[10] P. H. Eichel and C. V. Jakowatz, "Phase-gradient algorithm as an optimal estimator of the phase derivative," Opt. Lett., vol. 14, no. 20, pp. 11011103,1989

[11] J. A. Fawcett, "Inversion of N-dimensional spherical averages," Siam J. Math. Anal., vol. 45, no. 2, pp. 336-341, Apr. 1985.

[12] J. R. Fienup and J. J. Miller, "Aberration correction by maximizing generalized sharpness metrics," J. Opt. Soc., vol. 20, no. 4, pp. 609-620, Apr. 2003.

[13] P.-O. Frölind and L. M. H. Ulander, "Evaluation of angular interpolation kernels in fast factorized backprojection," Proc. Inst. Elect. Eng.-Radar, Sonar Navigat., vol. 153, no. 3, pp. 243-249, Jun. 2006.

[14] D. C. Ghiglia and G. A. Mastin, "Two-dimensional phase correction of synthetic-aperture-radar imagery," Opt. Lett., vol. 14, no. 20, pp. 1104 1106, Oct. 1989

[15] H. Hellsten and L-E. Andersson, "An inverse method for processing synthetic aperture radar data," Inv. Probl., vol. 3, no. 1, pp. 111-124, Feb. 1987

[16] H. Hellsten, P. Dammert, and A. Åhlander, "Autofocus in fast factorized backprojection for processing of SAR images when geometry parameters are unknown," in Proc. IEEE Radar Conf., 2010, pp. 603-608.

[17] H. Hellsten, J. Torgrimsson, P. Dammert, L. M. H. Ulander, and A. Åhlander, "Evaluation of a geometrical autofocus algorithm within the framework of fast factorized back-projection," in Proc. IET Radar, 2012, pp. $1-5$.

[18] H. Hellsten, L. M. H. Ulander, A. Gustavsson, and B. Larsson, "Development of VHF CARABAS II SAR," in Proc. SPIE Radar Sensor Technol., 1996, vol. 2747, pp. 48-60.

[19] C. V. Jakowatz and D. E. Wahl, "Eigenvector method for maximumlikelihood estimation of phase errors in synthetic-aperture-radar imagery," Opt. Lett., vol. 10, no. 12, pp. 2539-2546, Dec. 1993.

[20] C. V. Jakowatz, D. E. Wahl, P. H. Eichel, D. C. Ghiglia, and P. A. Thompson, Spotlight-Mode Synthetic Aperture Radar: A Signal Processing Approach. Norwell, MA, USA: Kluwer, 1996.

[21] J. Kolman, "PACE: An autofocus algorithm for SAR," in Proc. IEEE Int. Radar Conf., 2005, pp. 310-314.

[22] J. Kolman, "Continuous phase corrections applied to SAR imagery," in Proc. IEEE Radar Conf., 2009, pp. 1-5.

[23] A. Reigber, E. Alivizatos, A. Potsis, and A. Moreira, "Extended wavenumber-domain synthetic aperture radar focusing with integrated motion compensation," Proc. Inst. Elect. Eng.-Radar, Sonar Navigat., vol. 153 , no. 3 , pp. 301-310, Jun. 2006.

[24] F. Rocca, C. Cafforio, and C. Prati, "Synthetic aperture radar: A new application for wave equation techniques," Geophys. Prospecting, vol. 37, no. 7, pp. 809-830, Oct. 1989

[25] P. Samczynski and K. S. Kulpa, "Coherent mapdrift technique," IEEE Trans. Geosci. Remote Sens., vol. 48, no. 3, pp. 1505-1517, Mar. 2010.

[26] D. G. Thompson, J. S. Bates, D. V. Arnold, and D. G. Long, "Extending the phase gradient autofocus algorithm for low-altitude stripmap mode SAR," in Proc. IEEE Geosci. Remote Sens. Symp., 1999, pp. 564-566.

[27] L. M. H. Ulander, H. Hellsten, and G. Stenström, "Synthetic aperture radar processing using fast factorized backprojection," IEEE Trans. Aerosp. Electron. Syst., vol. 39, no. 3, pp. 760-776, Jul. 2003.

[28] V. T. Vu, T. K. Sjögren, and M. I. Pettersson, "On synthetic aperture radar azimuth and range resolution equations," IEEE Trans. Aerosp. Electron. Syst., vol. 48, no. 2, pp. 1764-1769, Apr. 2012.

[29] V. T. Vu, T. K. Sjögren, M. I. Pettersson, and H. Hellsten, "An impulse response function for evaluation of ultrawideband SAR imaging," IEEE Trans. Signal Process., vol. 58, no. 7, pp. 3927-3932, Jul. 2010.

[30] D. E. Wahl, "New approach to strip-map SAR autofocus," in Proc. IEEE 6th Digit. Signal Process. Workshop, 1994, pp. 53-56.

[31] D. E. Wahl, P. H. Eichel, D. C. Ghiglia, and C. V. Jakowatz, "Phase gradient autofocus-A robust tool for high resolution SAR phase correction," IEEE Trans. Aerosp. Electron. Syste., vol. 30, no. 3, pp. 827-835, Jul. 1994.

[32] D. W. Warner, D. C. Ghiglia, A. FitzGerrell, and J. Beaver, "Twodimensional phase gradient autofocus," in Proc. SPIE Image Reconstruction Incomplete Data, 2000, vol. 4123, pp. 162-173.

[33] A. F. Yegulalp, "Minimum entropy SAR autofocus," in Adaptive Sensor Array Processing (ASAP) Workshop, Lexington, MA, USA, 1999, MIT Lincoln Laboratory.
[34] L. Zhang, J. Sheng, M. Xing, Z. Qiao, T. Xiong, and Z. Bao, "Wavenumber-domain autofocusing for highly squinted UAV SAR imagery," IEEE Sensors J., vol. 12, no. 5, pp. 1574-1588, May 2012.

[35] D. Zhu, R. Jiang, X. Mao, and Z. Zhu, "Multi-subaperture PGA for SAR autofocusing," IEEE Trans. Aerosp. Electron. Syst., vol. 49, no. 1, pp. 468-488, Jan. 2013.

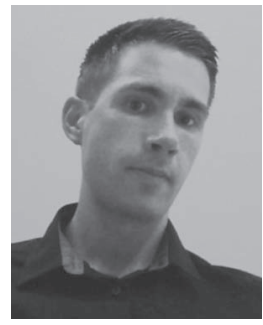

Jan Torgrimsson received the M.Sc. degree in engineering electronics from Umeå University, Umeå, Sweden, in 2009. He is currently working toward the $\mathrm{Ph} . \mathrm{D}$. degree in the Radar Remote Sensing Group, at Chalmers University of Technology, Gothenburg, Sweden.

His main research interest is synthetic aperture radar signal processing, with emphasis on image formation algorithms and autofocus.

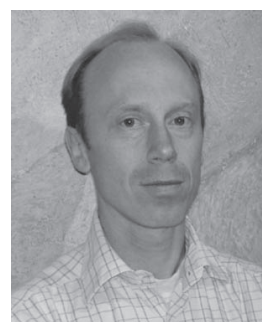

Patrik Dammert (S'95-M'00-SM'07) received the M.Sc. degree in electrical engineering and the $\mathrm{Ph} . \mathrm{D}$. degree in applications of spaceborne synthetic aperture radar (SAR) interferometry from Chalmers University of Technology, Gothenburg, Sweden, in 1993 and 1999, respectively.

From 1999 to 2000, he was a Research Assistant with Chalmers University of Technology. He later joined SAAB Electronic Defence Systems (formerly Ericsson Microwave Systems), Gothenburg, where he has been responsible for the development of high-performance SAR systems for airborne radars, spanning from VHF- to $\mathrm{X}$-band systems (with flat plate antennas and active electronically scanned array antennas). He has also been a Project Manager and an Associate Doctoral Student Supervisor for research projects with SAAB, in collaboration with Chalmers University of Technology and Blekinge Institute of Technology, Karlskrona, Sweden. His research interests are primarily in SAR systems, radar modeling, algorithms and signal processing, autofocus, and target detection in heavy-tailed radar clutter.

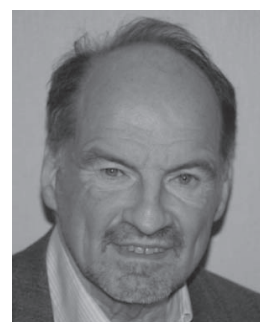

Hans Hellsten (M'06) received the Ph.D. degree in theoretical physics from Stockholm University, Stockholm, Sweden, in 1981.

He was a Research Director with the Swedish Defence Research Agency (FOI). He is currently a Senior Radar Expert with SAAB Electronic Defence Systems (EDS), Gothenburg, Sweden, where he also is the Head of the low-frequency radar product segment. Since the start of the low-frequency radar development in 1985, he has been the originator and one of the main developers of the technology, first at FOI and, since 2001, at SAAB EDS. In 1987, together with Lars-Erik Andersson, he formulated and solved for the first time the exact synthetic aperture radar reconstruction problem. Later on, he codeveloped with Lars Ulander the factorized back-projection method as practical realization of the exact solution. He is a holder of about 30 patents, which were granted in Sweden and other countries, mainly as the sole inventor. He has authored/coauthored numerous scientific and technical publications.

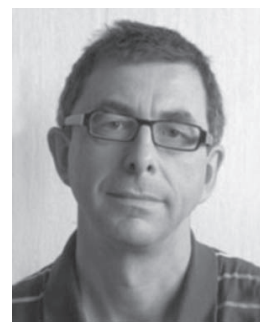

Lars M. H. Ulander (S'86-M'90-SM'04) received the M.Sc. degree in engineering physics and the $\mathrm{Ph} . \mathrm{D}$. degree in electrical and computer engineering from Chalmers University of Technology, Gothenburg, Sweden, in 1985 and 1991, respectively.

Since 1995, he has been with the Swedish Defence Research Agency (FOI), Linköping, Sweden, where he is the Director of research in radar signal processing and where he leads the research on very high frequency/ultrahigh frequency band radar. He is also currently an Adjunct Professor in radar remote sensing with Chalmers University of Technology. He has authored or coauthored over 300 professional publications, of which more than 50 are in peerreviewed scientific journals. He is a holder of five patents. His research areas are synthetic aperture radar, electromagnetic scattering models, and remote sensing applications 